\title{
Computational Investigation of the Spin-Density Asymmetry in Photosynthetic Reaction Center Models from First Principles
}

Denis G. Artiukhin ${ }^{\dagger *}$, Patrick Eschenbach", and Johannes Neugebauer ${ }^{\| *}$

† Department of Chemistry, Aarhus Universitet, DK-8000 Aarhus, Denmark

|| Theoretische Organische Chemie, Organisch-Chemisches Institut and Center for

Multiscale Theory and Simulation, Westfälische Wilhelms-Universität Münster, Corrensstraße 40, 48149 Münster, Germany

Date: $\quad$ March 29, 2020

*Email: artiukhin@chem.au.dk

*Email: j.neugebauer@uni-muenster.de 


\begin{abstract}
We present a computational analysis of the spin-density asymmetry in cation radical states of reaction center models from photosystem I, photosystem II, and bacteria from Synechococcus elongatus, Thermococcus vulcanus, and Rhodobacter sphaeroides, respectively. The recently developed FDE-diab methodology [J. Chem. Phys., 148 (2018), 214104] allowed us to effectively avoid the spin-density overdelocalization error characteristic for standard Kohn-Sham Density Functional Theory and to reliably calculate spin-density distributions and electronic couplings for a number of molecular systems ranging from inner pairs of (bacterio)chlorophyll $a$ molecules in vacuum to large protein including up to about 2000 atoms. The calculated spin densities show a good agreement with available experimental results and were used to validate reaction center models reported in the literature. Here we demonstrate that the applied theoretical approach is very sensitive to changes in molecular structures and relative orientation of molecules. This makes FDE-diab a valuable tool for electronic structure calculations of large photosynthetic models effectively complementing the existing experimental techniques.
\end{abstract}




\section{Introduction}

The mechanism of natural photosynthesis can conveniently be divided into several distinct steps [1]: i) light harvesting in antenna proteins, ii) a fast primary electron-transfer event in the reaction center $(\mathrm{RC})$, iii) protein stabilization due to charge separation, and iv) subsequent steps involving the synthesis of new chemical compounds. In the first step, the photosynthetic system does not undergo any chemical transformations and simply transfers the absorbed energy to the RC. The RC contains (bacterio)chlorophyll [(B)Chl] molecule(s), which constitute the primary electron donor. For the cases of green plants, algae, and cyanobacteria, two primary electron donors are known: P700 in photosystem I (PSI) and P680 in photosystem II (PSII) [1]. Bacterial reaction centers (BRCs) containing the electron donors P870 and P960 can be found in Rhodobacter sphaeroides and Rhodopseudomonas viridis, respectively [1]. Note that the numbers correspond to the absorption maxima of those complexes. The primary electron donors of PSI and the BRC are pairs of inner (B)Chl $a[2,3]$, which are called the special pair (SP) in cases of BRCs [4] (for an overview on RC structures, see Sec. 2). In case of PSII, the primary electron donor is composed of an accessory $\mathrm{Chl} a$ co-factor [3]. Being excited by the delivered energy, these primary electron donors donate an electron forming short-lived radical pairs with neighboring co-factors (for more detail, see Refs. [2,3]). In the third step, a chain of secondary electron-transfer reactions is initiated preventing the recombination of radical pairs back to neutral compounds and stabilizing the separation of charges. Thus, the absorbed energy is converted into electro-chemical potential, which is further used for synthesis of chemical compounds.

Although (B)Chl molecules in an inner pair are structurally very similar, they often have very different properties. The nearby protein environment could break the symmetry in the electronic structure of the pair, thus considerably changing its behavior during the electron donation. In case of a small asymmetry, this dimer could participate in electron- 
transfer processes as a supermolecule, whereas a strong asymmetry causes it to act as a pair of independent and weakly-coupled subunits. The degree of the electronic-structure asymmetry in inner pairs and corresponding radical cations, formed during the primary electron donation (for PSI and the BRC) or subsequent charge separation reactions (for PSII), is a frequently discussed issue in the field and received vast attention in the literature (for example, see Refs. [5-14]). In order to assess the asymmetry of the electronic ground state, chemical shifts of (B)Chl $a$ molecules are often considered [13,14], whereas spin-density distributions are used for cation radical states of inner pairs [6]. Solid-state Photochemically Induced Dynamic Nuclear Polarization Nuclear Magnetic Resonance (photo-CIDNP NMR) gives an access to both molecular properties. This methodology can be used to reconstruct spin-density maps of radical pairs inside RCs of plants and bacteria $[2,3]$ and can be applied to whole living cells [15] or even entire intact plants [16]. Another strategy is to access the $s$-orbital contributions to the spin density from measured hyperfine coupling constants (Fermi contact term) [17]. To that end, Electron Paramagnetic Resonance (EPR) and related methods such as Electron-Nuclear Double Resonance (ENDOR), Electron Spin Echo Envelope Modulation (ESEEM), and Electron-NuclearNuclear Triple Resonance (commonly referred to as TRIPLE) can be applied to single photosynthetic pigments [18-20] as well as to primary electron donors [21,22].

Theoretical studies are especially important for investigating the spin-density asymmetry as they provide a direct access to electronic structures of photosynthetic pigments and can be applied to obtain spin densities and hyperfine coupling constants of many shortlived open-shell intermediates produced in electron-transfer reactions. However, reliable calculations of inner pair spin-density distributions may require the consideration of the surrounding protein environment. This considerably increases the size of molecular models to be computed and restricts the choice of the electronic structure method to Kohn-Sham Density Function Theory (KS-DFT). The latter is affected by the self-interaction error (SIE) [23-25] and often produces overly delocalized spin-density distributions [26, 27]. 
This effect can be especially strong for non-covalently bonded molecular systems (for examples, see Refs. $[27,28])$ and, therefore, is expected to be crucial for pigments in RC models. Moreover, the degree of spin delocalization may strongly depend on the exchangecorrelation (XC) functional applied [29-32]. Although many different approaches were proposed in the literature to overcome this problem [27, 29-34], the ultimate solution is not yet found and the issue remains relevant.

One of these proposed solutions [27] is based on the use of the Frozen-Density Embedding (FDE) formalism [35] and allows to effectively avoid the spin-density overdelocalization error at the intermolecular region due to an embedding potential applied. This methodology seems especially attractive due to the possibility to consider much larger molecular systems than generally accessible with KS-DFT. However, its use leads to an opposite problem of overly localized spin densities. This drawback was recently lifted with FDE-diab [28], which extended and generalized the previously known Electron-Transfer Frozen-Density Embedding method (FDE-ET) [36-39]. The underlying idea of FDE-diab lies in the construction of charge- and spin-localized quasi-diabatic states and computing electronic couplings between them. A similar strategy was successfully applied in various electron-transfer calculations earlier [40-44] and proved to be very effective. The FDE-diab methodology was validated for a number of dimeric complexes featuring different degrees of the spin-density localization and was shown to be a more reliable and robust approach for spin-density calculations than standard KS-DFT [28]. In this work, we present FDE-diab calculations of spin densities and electronic couplings for RC models of PSI, PSII, and bacteria from Synechococcus elongatus, Thermococcus vulcanus, and Rhodobacter sphaeroides, respectively. We aim at reliable assessments of the spin-density asymmetry in inner pair radical cations and validation of previously proposed photosynthetic models. To that end, we consider a number of molecular systems ranging from dimeric complexes in vacuum to large protein and investigate roles of relative co-factor arrangements. 
In what follows, we briefly introduce RC architectures for PSI, PSII, and bacteria in Sec. 2. The underlying theory of the theoretical approach used is described in Sec. 3. In Sec. 4, we provide details on model setup and computations conducted followed by results presented in Sec. 5. Further discussions and conclusions are given in Sec. 6 .

\section{Architecture of Reaction Centers}

In the following, we limit our consideration to molecular structures of the RCs from PSII, PSI, and bacteria characteristical to the Thermococcus vulcanus, Synechococcus elongatus, and Rhodobacter sphaeroides photosynthetic organisms, respectively. The corresponding crystal structures were taken from Protein Data Bank (PDB) entries 3WU2 [45], 1JB0 [46], and 1M3X [47]. Similar RCs found in other organisms as well as mutants of the ones mentioned above are outside the scope of this paper and are not discussed here.

PSII is a protein complex located in the thylakoid membrane of oxygenic photosynthetic organisms. It is composed of two protein sub-units D1 and D2 (see Fig. 1). The membrane of PSII contains 36 trans-membrane $\alpha$-helices. Five of these helices belong to the proteins D1 and D2 each. Six of those belong to the proteins CP43 and CP47. CP43 and CP47 are called the core antenna proteins that bind 13 and $16 \mathrm{Chl}$ a molecules, respectively. Overall $11 \beta$-carotene units can be found in the antenna proteins. The central magnesium atom of the majority of the $\mathrm{Chl} a$ molecules in the antenna proteins is coordinated by a histidine (His) residue. Additionally, the tetrapyrrole macrocycle of these Chl $a$ molecules is not exactly planar, but slightly bent out of the macrocycle plane. The proteins D1 and D2 contain the co-factors that are associated with the RC of PSII. The D1 and D2 sub-units are distinguished by the presence of the so-called Oxygen Evolving Complex (OEC) near the sub-unit D1 (not shown in Fig. 1) and other structural aspects. The role of this manganese cluster is the oxidation of water and formation of oxygen [48]. 
The RC contains six Chl a molecules, of which four are believed to participate in the electron-transfer processes: i) the inner $\mathrm{P}_{\mathrm{D} 1}$ and $\mathrm{P}_{\mathrm{D} 2}$ co-factors composing the inner pair structurally similar to the SP in the BRC (depicted in green in Fig. 1) and ii) the accessory $\mathrm{Chl}_{\mathrm{D} 1}$ and $\mathrm{Chl}_{\mathrm{D} 2}$ pigments (blue). The $\mathrm{RC}$ also includes two Phe $a$ molecules dubbed Phe $\mathrm{D}_{\mathrm{D}}$ and $\mathrm{Phe}_{\mathrm{D} 2}$ (red) and two plastoquinone co-factors $\mathrm{Q}_{\mathrm{A}}$ and $\mathrm{Q}_{\mathrm{B}}$ (violet) as well as $\mathrm{Fe}(\mathrm{II})$ ion (brown) located near $\mathrm{Q}_{\mathrm{A}}$ and $\mathrm{Q}_{\mathrm{B}}$. The inner $\mathrm{P}_{\mathrm{D} 1 / \mathrm{D} 2}$ co-factors are coordinated by His residues, whereas the accessory $\mathrm{Chl}_{\mathrm{D} 1 / \mathrm{D} 2}$ pigments are coordinated by water molecules.

As seen from Fig. 1, PSI (just like PSII) also contains two protein sub-units, which are nearly $C_{2}$-symmetric. Its inner pair (shown in green) is formed by one Chl $a$ molecule and a $\mathrm{C}-13^{2}$-epimer of Chl $a$ (often referred to as Chl $a^{\prime}$ ), which are denoted as $\mathrm{P}_{\mathrm{B}}$ and $\mathrm{P}_{\mathrm{A}}$, respectively. It is well-known that $\mathrm{P}_{\mathrm{A}}$ forms several hydrogen bonds with amino acids from the surrounding protein environment, while $\mathrm{P}_{\mathrm{B}}$ does not $[46,49,50]$. The two accessory pigments are $\mathrm{Chl} a$ molecules and called $\mathrm{A}_{-1 \mathrm{~A}}$ and $\mathrm{A}_{-1 \mathrm{~B}}$ (blue). Similar to the case of PSII, the magnesium atoms of the inner co-factors $\mathrm{P}_{\mathrm{A}}$ and $\mathrm{P}_{\mathrm{B}}$ are coordinated by His molecules, whereas the magnesium atoms of accessory pigments $\mathrm{A}_{-1 \mathrm{~A}}$ and $\mathrm{A}_{-1 \mathrm{~B}}$ are coordinated by water molecules. Instead of Phe $a$ molecules, the RC of PSI contains two Chl $a$ co-factors, which are referred to as $\mathrm{A}_{0 \mathrm{~A}}$ and $\mathrm{A}_{0 \mathrm{~B}}$ (red) and feature the central magnesium atoms being coordinated by sulfur atoms of methionine residues. In addition to the above-mentioned pigments, this $\mathrm{RC}$ also includes two phylloquinone co-factors $\mathrm{A}_{1 \mathrm{~A}}$ and $\mathrm{A}_{1 \mathrm{~B}}$ (violet) and an $[4 \mathrm{Fe}-4 \mathrm{~S}]$ cluster $\mathrm{F}_{\mathrm{x}}$ (brown).

In the $\mathrm{BRC}$, the $\mathrm{SP}$ of pigments is formed by $\mathrm{BChl} a$ molecules, which are denoted as $\mathrm{D}_{\mathrm{A}}$ and $\mathrm{D}_{\mathrm{B}}$ (green). Similar BChl a molecules compose the pair of accessory co-factors $\mathrm{B}_{\mathrm{A}}$ and $\mathrm{B}_{\mathrm{B}}$ (blue). It is interesting to note that the central magnesium atoms of these four pigments are all coordinated by axial His molecules. In contrast to the case of PSII, the BRC features a pair of BPhe $a$ molecules instead of Phe $a$, which are called $\mathrm{H}_{\mathrm{A}}$ and $\mathrm{H}_{\mathrm{B}}$ and 
depicted in red in Fig. 1. The pair of quinone molecules is represented by menaquinone $\mathrm{Q}_{\mathrm{A}}$ and ubiquinone $\mathrm{Q}_{\mathrm{B}}$. Additionally, the BRC contains, just like in PSII, a Fe(II) ion (brown) located between the $\mathrm{Q}_{\mathrm{A}}$ and $\mathrm{Q}_{\mathrm{B}}$ co-factors. 

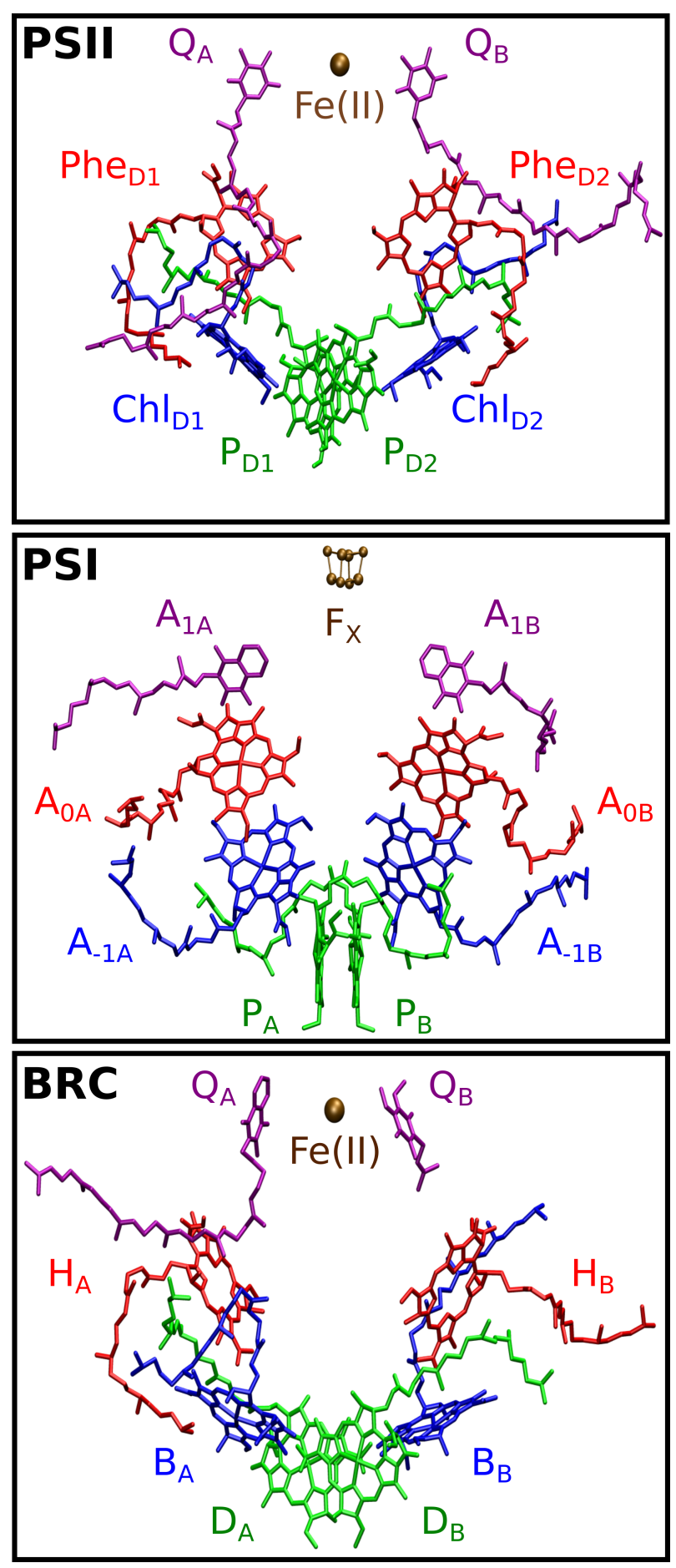

Figure 1: Graphical representation of the structural arrangement of the co-factors in photosynthetic RCs of PSII, PSI, and bacteria. The crystal structures are taken from PDB entries 3WU2 [45], 1JB0 [46], and 1M3X [47]. For the color code used, see the main text. 


\section{Theory}

The underlying theory of the FDE-diab approach [28,36-39] will be briefly repeated here for the specific case of two non-covalently bound molecules $A$ and $B$ forming a radical cation complex $[A \cdots B]^{+\bullet}$. This rather simple consideration has a direct connection to CT processes often occurring between two pigments in photosynthetic systems and is sufficient enough for dimeric molecular models presented in this work. Note, however, that generalization to multiple fragments is straightforward from a mathematical point of view. In these radical cation complexes, charge and spin are delocalized to some extent between both molecules. The spin-density ratio can range from a complete delocalization (i.e., $50 \% / 50 \%$ ) to a localization at only one fragment. The former case can be found in structurally symmetric dimers $A_{2}{ }^{+\bullet}$, while the latter case is characteristic for fragments with different ionization energies and large intermolecular separations. As was mentioned above, a description of the radical cation electronic structure in general and prediction of spin-density delocalizations in particular may be problematic with both the KS-DFT and FDE approaches. However, the tendency of FDE to overlocalize charge and spin density can be used to construct an approximate electronic wave function $\Psi$ of the radical cation complex $[A \cdots B]^{+\bullet}$, which provides a qualitatively correct description of the electronic structure. This can be achieved by computing two wave functions $\Phi_{1}$ and $\Phi_{2}$ corresponding to the charge-localized states $\left|A^{+\bullet} B\right\rangle$ and $\left|A B^{+\bullet}\right\rangle$ and taking the total electronic wave function $\Psi$ as a linear combination,

$$
\Psi=a \Phi_{1}+b \Phi_{2}
$$

with linear combination coefficients $a$ and $b$. This basis of two quasi-diabatic wave functions $\Phi_{1}$ and $\Phi_{2}$ can then be used to evaluate the elements of overlap and Hamilton matrices to subsequently solve a generalized eigenvalue problem. The resulting eigenvector elements $a$ and $b$ define the total wave function $\Psi$ and, thus, give an access to calculations of various molecular properties of the radical cation complex $[A \cdots B]^{+\bullet}$. In 
the following sections, we briefly describe the FDE-diab methodology and show how accurate electronic couplings, long-range excitations, and spin densities can be calculated from the total wave function.

\subsection{Construction of Charge-Localized States}

In order to construct the required wave functions $\Phi_{1}$ and $\Phi_{2}$, we carry out FDE [35] computations of the radical cations $\left[A^{+\bullet} \cdots B\right]$ and $\left[A \cdots B^{+\bullet}\right]$ with positive charges localized at the molecules $A$ and $B$, respectively. In these calculations, sets of molecular orbitals (MOs) $\psi_{i}^{I, \sigma}(\vec{r})$ of separate subsystems $A, B, A^{+\bullet}$, and $B^{+\bullet}$ and their corresponding energies $\epsilon_{i}^{I, \sigma}$ are obtained from the so-called Kohn-Sham equations with constrained electronic density (KSCED) [35, 51],

$$
\left[-\frac{\nabla^{2}}{2}+v_{\mathrm{KS}}^{I, \sigma}(\vec{r})+v_{\mathrm{emb}}^{I, \sigma}(\vec{r})\right] \psi_{i}^{I, \sigma}(\vec{r})=\epsilon_{i}^{I, \sigma} \psi_{i}^{I, \sigma}(\vec{r}),
$$

where $-\frac{\nabla^{2}}{2}$ is the one-electron kinetic energy operator, while $v_{\mathrm{KS}}^{I, \sigma}(\vec{r})$ and $v_{\mathrm{emb}}^{I, \sigma}(\vec{r})$ are the one-electron KS and embedding potential, respectively. The superscript $I$ denotes the subsystem under study and $\sigma=\alpha$ or $\beta$ are used as spin labels. The charge-localized states $\Phi_{1}$ and $\Phi_{2}$ are then constructed as direct products $[43,52]$,

$$
\Phi_{1} \equiv\left|A^{+\bullet} B\right\rangle=\left|A^{+\bullet}\right\rangle \otimes|B\rangle
$$

and

$$
\Phi_{2} \equiv\left|A B^{+\bullet}\right\rangle=|A\rangle \otimes\left|B^{+\bullet}\right\rangle
$$

of KS determinants $|A\rangle,|B\rangle,\left|A^{+\bullet}\right\rangle$, and $\left|B^{+\bullet}\right\rangle$. The resulting functions $\Phi_{1}$ and $\Phi_{2}$ are, therefore, Slater determinants composed of subsystem orbitals. Such a use of KS-like MOs in $\Phi_{1}$ and $\Phi_{2}$ is a common approximation often applied in the literature for CT simulations [52-54]. If a monomer basis set is used in FDE calculations, the resulting 
matrices of $\mathrm{MO}$ coefficients for $\Phi_{1}$ and $\Phi_{2}$ take block-diagonal forms and are given by

$$
\mathrm{C}_{\Phi_{1}}=\left(\begin{array}{cc}
\mathrm{C}_{A^{+}} & \mathbf{0} \\
\mathbf{0} & \mathrm{C}_{B}
\end{array}\right)
$$

and

$$
\mathrm{C}_{\Phi_{2}}=\left(\begin{array}{cc}
\mathrm{C}_{A} & \mathbf{0} \\
\mathbf{0} & \mathrm{C}_{B^{+}}
\end{array}\right) .
$$

The use of this monomer basis set is advantageous for large molecular systems as it allows for considerable savings in the overall computational cost. It should be noted that the KS-like orbitals forming Slater determinants of the charge-localized states $\Phi_{1}$ and $\Phi_{2}$, are in general not orthogonal, which leads to non-orthogonality of these states. The overlaps for the latter are given by $[55,56]$

$$
S_{n m}=\left\langle\Phi_{n} \mid \Phi_{m}\right\rangle=\operatorname{det}\left(\mathbf{S}^{(n m)}\right)
$$

where $\operatorname{det}\left(\mathbf{S}^{(n m)}\right)$ is a determinant of the overlap matrix $\mathbf{S}^{(n m)}$, which consists of MO overlaps $S_{i j}^{(n m)}=\left\langle\psi_{i}^{(n), \sigma} \mid \psi_{j}^{(m), \sigma}\right\rangle$. It should also be noted that the constructed chargelocalized states $\Phi_{1}$ and $\Phi_{2}$ are not necessarily normalized.

Alternatively, charge localized states can be constructed with, e.g., constrained Density Functional Theory (CDFT) [57]. This approach, however, requires the use of rather hard constraints on the electronic density and is computationally more expensive than FDE. The latter introduces only soft "restraints" to the subsystems electronic densities and provides the possibility for their easier mutual polarization by means of freeze-and-thaw cycles [58]. 


\subsection{Solution of the Generalized Eigenvalue Problem}

To find the unknown coefficients $a$ and $b$ and obtain the total wave function $\Psi$ from Eq. (1), the generalized eigenvalue problem,

$$
\mathbf{H c}=E \mathbf{S c},
$$

needs to be solved in the basis of two charge-localized states $\left\{\Phi_{1}, \Phi_{2}\right\}$. Here, $\mathbf{H}$ and $\mathbf{S}$ are the $2 \times 2$ matrices of the Hamiltonian and overlap, respectively, whereas $\mathbf{c}$ is a column vector of the linear combination coefficients. The elements $S_{n m}$ of the overlap matrix are given according to Eq. (7), whereas the elements of the Hamiltonian are calculated approximately as functionals of the transition electronic densities $\rho_{n m}(\vec{r})=\rho_{n m}^{\alpha}(\vec{r})+$ $\rho_{n m}^{\beta}(\vec{r})$, scaled by the overlap $S_{n m}[57,59]$,

$$
H_{n m}=\left\langle\Phi_{n}|\hat{H}| \Phi_{m}\right\rangle \approx E\left[\frac{\rho_{n m}^{\alpha}(\vec{r})+\rho_{n m}^{\beta}(\vec{r})}{S_{n m}}\right] S_{n m} .
$$

This approximation is invoked to obtain both diagonal and off-diagonal matrix elements in a consistent way using approximate exchange-correlation (XC) functionals (for more details, see Ref. [37]). The $\alpha$ - and $\beta$-components of the electronic transition density $\rho_{n m}(\vec{r})$ can be obtained as eigenvalues of the one-electron $\sigma$-density operator $\hat{\rho}^{\sigma}$,

$$
\rho_{(n m)}^{\sigma}(\vec{r})=\left\langle\Phi_{n}\left|\hat{\rho}^{\sigma}\right| \Phi_{m}\right\rangle=S_{n m} \sum_{i, j=1}^{N_{\sigma}} \psi_{i}^{(n), \sigma} \psi_{j}^{(m), \sigma}\left(\mathbf{S}^{(n m), \sigma}\right)_{j i}^{-1},
$$

with $\sigma=\alpha$ or $\beta$. Here, the summation on the right-hand side runs over the $N_{\sigma} \sigma$ -

electrons in the radical cation and includes products of spin-orbitals $\psi_{i}^{(n), \sigma}$ with the elements $\left(\mathbf{S}^{(n m), \sigma}\right)_{j i}^{-1}$ of the corresponding transposed inverse overlap matrix $\left[\left(\mathbf{S}^{(n m), \sigma}\right)^{-1}\right]^{\mathrm{T}}$. For a more detailed derivation of this expression, we refer the reader to our previous work [28]. With the elements of the Hamiltonian and the overlap matrix obtained, we can write the secular determinant,

$$
\operatorname{det}(\mathbf{H}-E \mathbf{S})=\left|\begin{array}{ll}
H_{11}-E S_{11} & H_{12}-E S_{12} \\
H_{12}-E S_{12} & H_{22}-E S_{22}
\end{array}\right|=0,
$$


and solve the generalized eigenvalue problem from Eq. (8). This gives us energies $E_{0}$ and $E_{1}$ of the resulting ground $\left(\Psi_{0}\right)$ and excited $\left(\Psi_{1}\right)$ state with the corresponding sets of linear combination coefficients $\left\{a_{0}, b_{0}\right\}$ and $\left\{a_{1}, b_{1}\right\}$.

FDE-ET [36-39] introduces an additional approximation to the generalized eigenvalue problem described above enabling the use of an analytical expression for electronic couplings. This can be achieved by i) assuming that $S_{11}=S_{22}$, ii) dividing the off-diagonal element $S_{12}$ by $\sqrt{S_{11} S_{22}}$ (to obtain $S_{12}^{\prime}$ ), and iii) omitting the term $S_{n n}$ on the right-hand side of Eq. (9) for the case of diagonal elements such that

$$
H_{n n}^{\prime}=E\left[\frac{\rho_{n n}^{\alpha}(\vec{r})+\rho_{n n}^{\beta}(\vec{r})}{S_{n n}}\right]
$$

In this case, the secular determinant takes the simplified form

$$
\operatorname{det}(\mathbf{H}-E \mathbf{S})=\left|\begin{array}{cc}
H_{11}^{\prime}-E & H_{12}-E S_{12}^{\prime} \\
H_{12}-E S_{12}^{\prime} & H_{22}^{\prime}-E
\end{array}\right|=0
$$

and can be solved analytically yielding two eigenvalues (say $E_{0}$ and $E_{1}$ ) with the energy difference $\Delta E$ given by the expression $[42,43,60]$

$$
\Delta E=\sqrt{\frac{\left(H_{11}^{\prime}-H_{22}^{\prime}\right)^{2}}{1-S_{12}^{\prime 2}}+4 V_{12}^{2}} .
$$

Here, $V_{12}$ is equal to

$$
V_{12}=\frac{1}{1-S_{12}^{\prime 2}}\left[H_{12}-S_{12}^{\prime} \frac{H_{11}^{\prime}+H_{22}^{\prime}}{2}\right]
$$

The terms $\Delta E$ and $V_{12}$ are often referred to as excitation energy and electronic coupling, respectively. This approximation has proven to be sufficiently accurate for modeling CT reactions and calculations of long-range vertical excitation energies (for a benchmark study on the hole transfer, see Ref. [39]). 


\subsection{Electronic and Spin-Density Distributions}

In our previous study in Ref. [28], we outlined in very detail how the spin density $\rho^{\alpha-\beta}(\vec{r})$ can be obtained from the total wave function $\Psi$. The idea behind this is rather simple and is based on the direct evaluations of the $\alpha$ - and $\beta$-density contributions as eigenvalues of $\hat{\rho}^{\sigma}$,

$$
\begin{gathered}
\rho^{\sigma}(\vec{r})=\frac{\left\langle\Psi\left|\hat{\rho}^{\sigma}\right| \Psi\right\rangle}{\langle\Psi \mid \Psi\rangle}=\frac{a^{2}\left\langle\Phi_{1}\left|\hat{\rho}^{\sigma}\right| \Phi_{1}\right\rangle+2 a b\left\langle\Phi_{1}\left|\hat{\rho}^{\sigma}\right| \Phi_{2}\right\rangle+b^{2}\left\langle\Phi_{2}\left|\hat{\rho}^{\sigma}\right| \Phi_{2}\right\rangle}{a^{2}\left\langle\Phi_{1} \mid \Phi_{1}\right\rangle+2 a b\left\langle\Phi_{1} \mid \Phi_{2}\right\rangle+b^{2}\left\langle\Phi_{2} \mid \Phi_{2}\right\rangle} \\
=\frac{a^{2} \rho_{(11)}^{\sigma}(\vec{r})+2 a b \rho_{(12)}^{\sigma}(\vec{r})+b^{2} \rho_{(22)}^{\sigma}(\vec{r})}{a^{2} S_{11}+2 a b S_{12}+b^{2} S_{22}}
\end{gathered}
$$

where transition densities $\rho_{(n m)}^{\sigma}(\vec{r})$ are given by Eq. (10). The spin-density function $\rho^{\alpha-\beta}(\vec{r})$ is then calculated as the difference between $\alpha$ - and $\beta$-components,

$$
\rho^{\alpha-\beta}(\vec{r})=\rho^{\alpha}(\vec{r})-\rho^{\beta}(\vec{r})
$$

Analogously, the total electronic density $\rho(\vec{r})$ of the molecular system can be obtained as the sum of $\alpha$ - and $\beta$-densities,

$$
\rho(\vec{r})=\rho^{\alpha}(\vec{r})+\rho^{\beta}(\vec{r})
$$

from Eq. (16). This rather trivial fact has, however, a very large importance as it allows for DFT-type calculations of various molecular properties that are derived from the spin density, e.g. hyperfine coupling constants, with potentially higher quality than those from conventional KS-DFT. The higher quality of FDE-diab spin densities compared to those of conventional KS-DFT was already validated in previous work [28]. Whether this also holds for spin density derived properties remains to be tested.

\section{Computational Details}

Molecular models for PSI, PSII, and the BRC were extracted from crystal structures of Synechococcus elongatus [46] (PDB entry 1JB0), Thermococcus vulcanus [45] (PDB en- 
try 3WU2), and Rhodobacter sphaeroides [47] (PDB entry 1M3X), respectively, obtained from the Protein Data Bank [61]. Missing hydrogen atoms of photosynthetic co-factors were added with the REDUCE program [62], whereas the hydrogen atoms of the protein environment were added using CHARMM22 topology files [63,64]. To saturate cut protein bonds, neutral groups $-\mathrm{C}(\mathrm{O}) \mathrm{CH}_{3}$ and $-\mathrm{NH}_{2}$ were applied as $\mathrm{N}$ - and C-termini, respectively. In these capping groups, bond distances were initially set to experimentally determined average values characteristic for small organic compounds [65]. Two types of models were considered in this work: i) models, in which the long hydrocarbon tails $\mathrm{R}$ of Chl molecules (see Fig. 2) were left unchanged and ii) models with the tail $\mathrm{R}$ truncated and replaced by a $-\mathrm{CH}_{3}$ group. In case of dimeric molecular models such as the inner pair, sets of modified molecular structures with different orientations of the fragments were created. Thus, the initial crystal structures were adjusted by making mutual vertical and horizontal shifts of chlorophyll molecules. For this purpose, coordinates of truncated chlorophyll rings (without hydrogens and substituents at aromatic cycles) were fitted by a pair of parallel planes minimizing vertical distances to the atoms. Then, vectors of perpendicular and parallel shifts were determined. For the latter case, we chose vectors lying within the parallel planes and oriented along the $\mathrm{Mg} \rightarrow \mathrm{C}-5$ directions of the corresponding molecules. In the following, we denote distances between these parallel planes as $R_{\perp}$, while vertical and horizontal shifts are referred to as $\Delta R_{\perp}$ and $\Delta R_{\|}$, respectively. Note that in case of horizontal shifts $\Delta R_{\|}$, coordinates of both aromatic macro-cycles are modified simultaneously by the corresponsing translation vectors of lengths $\Delta R_{\|}$. Binding pocket models of PSI, PSII, and BRC considered in Sec. 5.3 were created by specifying radii of $4.0 \AA$ around each atom of the inner pair. All surrounding co-factors, water molecules, and amino acid residues with at least one atom within these radii were included explicitly into the models. Addition of missing hydrogens and cut bond saturation were conducted in a similar fashion as for smaller models considered in this work (see above). Amino acid residues were considered to be neutral and protonated in the created binding pocket 
models.

Chl a

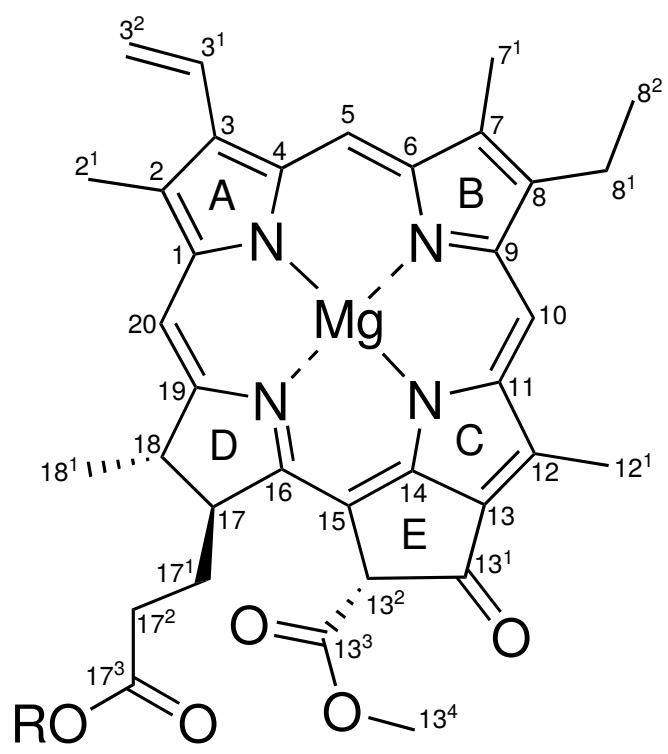

$\mathrm{BChl} a$

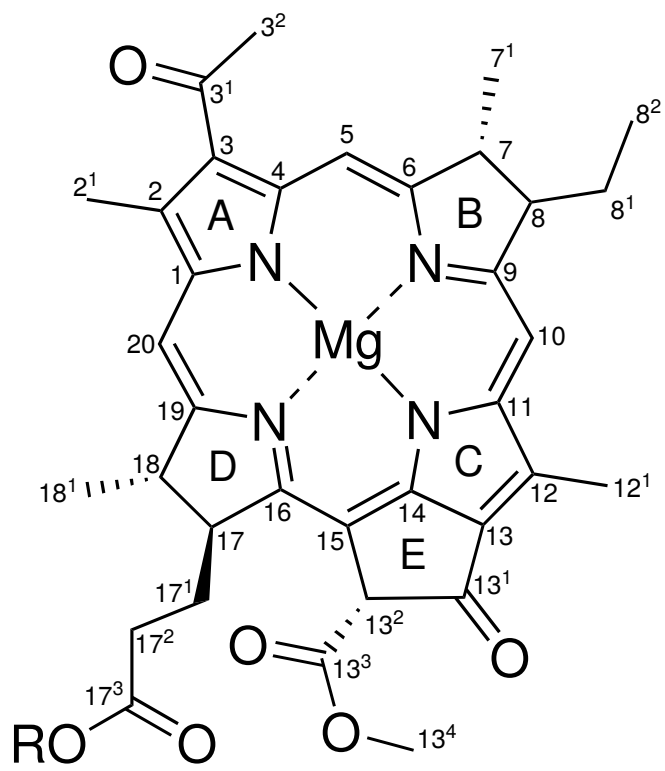

$\mathrm{R}=\mathrm{C}_{\mathrm{P}^{2}}^{\mathrm{P}^{1}}$

Figure 2: Lewis structures of $\mathrm{Chl} a$ and $\mathrm{BChl} a$. The atomic numbering is given according to IUPAC.

To distinguish the created inner pair models with and without phytyl tails, we introduce label "t" for truncation. For example, $\left[\mathrm{tP}_{\mathrm{D} 1} \cdots \mathrm{tP}_{\mathrm{D} 2}\right]^{+\bullet}$ should be understood as the truncated complex of $\mathrm{P}_{\mathrm{D} 1}$ and $\mathrm{P}_{\mathrm{D} 2}$, while its non-truncated counterpart is denoted as $\left[\mathrm{P}_{\mathrm{D} 1} \cdots \mathrm{P}_{\mathrm{D} 2}\right]^{+\bullet}$. For comparison purposes, similar models are considered for the inner co-factors $\mathrm{P}_{\mathrm{A}}=$ Chl $a^{\prime}$ and $\mathrm{P}_{\mathrm{B}}=$ Chl $a$ from the RC of PSI and for $\mathrm{D}_{\mathrm{A}}=\mathrm{BChl} a$ of the BRC.

Created molecular structures for neutral dimeric models in vacuum were optimized in two subsequent steps using the ORCA [66] program package. At first, the positions of hydrogens were relaxed keeping all other atomic coordinates fixed. Secondly, the structure 
optimization was carried out for the set of intramolecular bond distances. Two atoms $A$ and $B$ were considered as bound if the distance between them is in the interval of $r(A)+r(B) \pm t$, where $r(A)$ and $r(B)$ are covalent radii of the corresponding atoms $[67,68]$ and $t=0.5 \AA$ is a tolerance parameter. This procedure ensured that structural parameters are partly optimized, while the relative orientation of fragments is still very close to that in the original crystal structure. Note that the second step does not imply any explicit constraints to bond and dihedral angles. In fact, they can slightly change during the optimization procedure. In both steps, the BP86 XC functional $[69,70]$ and the def2TZVP basis set $[71,72]$ were applied. To reduce the computational cost, the resolution-ofthe-identity approximation in conjunction with the auxiliary Coulomb-fitting def2-TZV/J basis $[71,72]$ was enabled. Dispersion interactions were taken into account with the D3BJ correction with Becke--Johnson damping [73,74]. "Loose" convergence criteria were applied for inner pair models. These optimized molecular structures of neutral complexes were then used in subsequent single-point calculations of the corresponding radicals.

For geometry optimizations of binding pockets as described in Sec. 5.3, the DFTB3 [75] method within the AMs-DFTB module from the ADF 2019 package [76] was used. The Third-Order Parametrization for Organic and Biological Systems (3ob) [77,78] parameters from the corresponding Slater-Koster file were used. Dispersion interactions were taken into account with the D3BJ correction with Becke--Johnson damping $[73,74]$. The optimization was carried out in two consecutive steps. In the first step, the coordinates corresponding to hydrogen atoms were optimized, while all other nuclear positions were kept fix. In the second step, only the nuclear coordinates corresponding to the inner pair were optimized. This procedure ensures to keep the relative arrangement of environment residues and co-factors unchanged, while the inner pair coordinates are relaxed in the presence of the surrounding pocket. Default convergence criteria were applied for the optimization of hydrogen positions, whereas "loose" criteria were used in the second step of geometry optimization. 
For FDE-diab and FDE-ET computations, locally modified versions of the ADF program [76] and the PYADF scripting framework [79] were used. All calculations employed the TZP basis set [80] from the ADF program library and the PW91 [81,82] XC functional with the conjoint [83] kinetic-energy functional PW91k [84]. Mutual relaxations of subsystem densities were taken into account applying freeze-and-thaw cycles [58]. Three such cycles were found sufficient to obtain accurate electronic densities (see also Ref. [28]). In FDE-diab and FDE-ET calculations of dimeric systems such as inner pair models, KSlike MOs of both molecules were used for the construction of diabatic states. For binding pocket models, MOs of only inner pair Chls were explicitly included into the FDE-diab step, while interactions with MOs of environment molecules were taken into account by means of orbital polarization during preceding freeze-and-thaw cycles.

To provide a quantitative measure for spin-density delocalizations, a Becke-type population analysis was employed as was done in previous works $[28,85]$. For this purpose, accurate atom-centered Becke grids [86,87] were generated using the SERENITY program [88]. During this procedure, grid points were assigned to particular nuclei forming overlapping atomic basins. Spin-density values at grid points were extracted with the DENSF program from the ADF package and then integrated over the generated basins giving atomic spin-density populations. The reported spin-density delocalization ratios were obtained by summing up these atomic contributions for the corresponding molecules. The error introduced by numerical integration was found to be below $5.0 \times 10^{-4}$ a.u. in all cases studied. For comparison of this integration scheme with standard Bader and Mulliken population analysis, see Sec. S3 in the Supporting Information (SI). 


\section{Results}

In Sec. 5.1 we provide a detailed explanation of spin densities obtained within the two-state model for a better understanding of the following result sections. In Sec. 5.2, physically motivated dimeric molecular models for the electronic donors of PSI, PSII, and BRCs are calculated in vacuum. The influence of the protein environment is then considered in Sec. 5.3.

\subsection{Spin Densities from the Two-State Model}

The use of the FDE-diab approach within the two-state model allows for an easy interpretation of the resulting spin-density distribution. The qualitative character of this distribution can be fully predicted based on the absolute value of the electronic coupling $\left|V_{12}\right|$ and the energy difference $H_{11}^{\prime}-H_{22}^{\prime}$ between quasi-diabatic states. Depending on these values, two different scenarios can be distinguished (see Fig. 3). If $\left|V_{12}\right|$ and $\left|H_{11}^{\prime}-H_{22}^{\prime}\right|$ are comparable in magnitude, coupling of charge-localized electronic states $\Phi_{1}$ and $\Phi_{2}$ occurs (Fig. 3, left). This gives rise to the two adiabatic electronic wave functions $\Psi_{0}$ and $\Psi_{1}$ with the corresponding energies $E_{0}$ and $E_{1}$. In this case, the excitation energy $\Delta E=E_{1}-E_{0}$ is considerably larger than the original energy gap $\left|H_{11}^{\prime}-H_{22}^{\prime}\right|$. The resulting electronic states $\Psi_{0}$ and $\Psi_{1}$ are written as linear combinations,

$$
\Psi_{0}=a_{0} \Phi_{1}+b_{0} \Phi_{2}, \quad \Psi_{1}=a_{1} \Phi_{1}+b_{1} \Phi_{2}
$$

where linear coefficients $a_{i}$ and $b_{j}$ are all significantly different from zero. Because the initial states $\Phi_{1}$ and $\Phi_{2}$ are charge- and spin-localized, the spin-density distributions corresponding to the linear combinations $\Psi_{0}$ and $\Psi_{1}$ are delocalized. The degree of such delocalization depends on the linear combination coefficients $a_{0}, b_{0}$ and $a_{1}, b_{1}$ and grows with the electronic coupling $\left|V_{12}\right|$. 


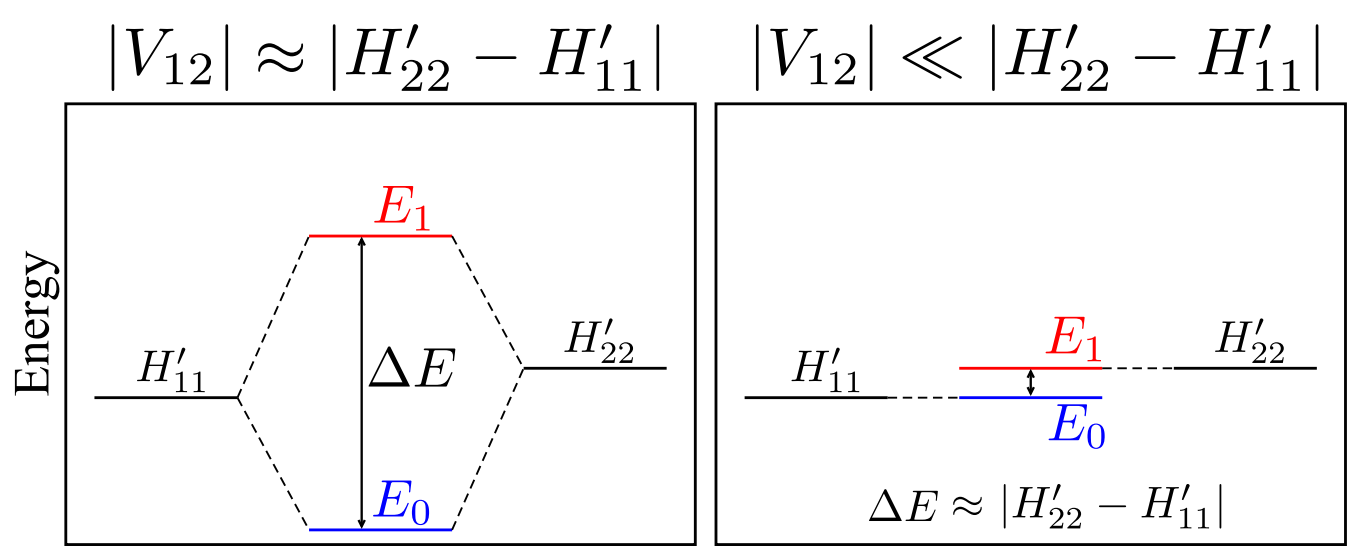

Figure 3: Schematic representation of the two possible coupling scenarios for the quasidiabatic states $\Phi_{1}$ and $\Phi_{2}$. See main text for further discription.

The second scenario occurs if $\left|V_{12}\right|$ is much smaller than $\left|H_{11}^{\prime}-H_{22}^{\prime}\right|$ (see Fig. 3, right). In this case, the quasi-diabatic electronic states do not couple significantly and result in almost unchanged energy values $E_{0} \approx H_{11}^{\prime}$ and $E_{1} \approx H_{22}^{\prime}$. The adiabatic wave functions $\Psi_{0}$ and $\Psi_{1}$ are, thus, dominated by single Slater determinants and are approximately written as,

$$
\Psi_{0} \approx a_{0} \Phi_{1}, \quad \Psi_{1} \approx b_{1} \Phi_{2}
$$

with the coefficients $b_{0}$ and $a_{1}$ being very close to zero. Therefore, the spin-density distributions are calculated from single charge-localized determinants $\Phi_{1}$ and $\Phi_{2}$ and are completely localized at particular molecular fragments.

All FDE-diab spin-density distributions considered below can be interpreted within the two coupling scenarios described in this section.

\subsection{The Inner Pair}

Although the spatial arrangement of (B)Chl molecules in the inner pairs of PSI, PSII, and BRC is very similar in all three cases (see Fig. 1), small structural differences occur. The first important difference to be mentioned is the spatial gap between the co-factors 
composing the inner pair. If we define this gap through the distance $R_{\perp}$ between parallel planes going through the aromatic macrocycles of the co-factors (for a more robust definition of these planes, see Sec. 4), then the largest separation between inner (B)Chl molecules can be found in the crystal structure of PSI [46] and is equal to about $3.9 \AA$. A slightly smaller value of $3.7 \AA$ can be found in case of PSII [45]. The SP of the BRC features the smallest distance $R_{\perp}=3.1 \AA$ between the co-factors [47]. Therefore, one might expect that the interaction energy between the co-factors is the largest for the $\left[D_{A} \cdots D_{B}\right]$ pair in the BRC and that it decreases for the inner pairs in the following order: BRC, PSII, PSI. The second distinction that can be identified by a close analysis of the crystal structures is the degree to which the aromatic macrocycles overlap. Thus, the inner pairs of PSII and BRC are arranged such that the substituents $-\mathrm{CH}_{2}=\mathrm{CH}_{2}$ and $-\mathrm{C}(\mathrm{O})-\mathrm{CH}_{3}$ (for $\mathrm{Chl} a$ of PSII and BChl $a$ of BRC, respectively) at carbon atom C-3 of one co-factor are almost directly above the $\mathrm{Mg}$ atom of the second co-factor. The inner pair in PSI shows much larger overlap with the corresponding group $-\mathrm{CH}_{2}=\mathrm{CH}_{2}$ shifted further towards carbon atom $\mathrm{C}-15$. It is also interesting to note that the $\mathrm{BChl} a$ macrocycles in the crystal structure of BRC are essentially planar, while a strong out-of-plane bent is present for both PSI and PSII inner pairs. These spatial arrangements of co-factors are largely governed by interactions with the surrounding protein pockets, which are often arranged in an asymmetrical way around the constituting inner pair molecules and interact differently with those. Thus, the inner co-factors of PSII interact strongly with the surrounding protein, whereas $\mathrm{P}_{\mathrm{A}}$ and $\mathrm{P}_{\mathrm{B}}$ are reported to be essentially undisturbed [89]. It is also well known that $\mathrm{P}_{\mathrm{A}}$ of PSI forms hydrogen bonds with several amino acids from the surrounding protein, whereas $\mathrm{P}_{\mathrm{B}}$ does not $[46,49,50]$. In case of PSII, the spin-density distribution of the inner pair was experimentally obtained with ENDOR spectroscopy [8]. These experimental measurements predicted $82 \%$ of the unpaired spin to be localized at the $\mathrm{P}_{\mathrm{D} 1}$ monomer, while the $18 \%$ left were assigned to $\mathrm{P}_{\mathrm{D} 2}$. The authors of Ref. [90] conducted a ${ }^{13} \mathrm{C}$ photo-CIDNP MAS NMR experiment assigning most of the measured signals 
to a single Chl $a$ co-factor. The observed spin-density distribution showed a pronounced asymmetry (shift towards rings III and V) within Chl $a$ compared to the results obtained in solution (largest contribution at ring II) [20]. This asymmetry was later confirmed in a subsequent experiment [89]. Finally, ${ }^{15} \mathrm{~N}$ photo-CIDNP MAS NMR measurements carried out in Ref. [91] detected signals originating from an axial histidine molecule leading to the formulation of the hinge-type model for the primary electron donor of PSII. Therefore, according to the experimental results the inner pair of PSII is a weakly-coupled pair with the largest spin-density contribution localized at the $\mathrm{P}_{\mathrm{D} 1}$ co-factor. The remaining spin-density is probably localized at the axial His molecule or shared between His and $\mathrm{P}_{\mathrm{D} 2}$.

Early EPR [92] and ENDOR [93] measurements of PSI reported a fully symmetrical spindensity distribution between both monomers in P700. However, more recent work [7-10, 12] proposed a large range of asymmetric spin-density distributions ranging from $75 \% / 25 \%$ to about $91 \% / 9 \%$ in favor of $\mathrm{P}_{\mathrm{B}}$ and even completely localized at $\mathrm{P}_{\mathrm{B}}$ distributions. Thus, ESEEM experiments by Davis et al. [7] proposed that the inner pair in spinach PSI is either a dimer with the spin-density ratio from $75 \% / 25 \%$ to $80 \% / 20 \%$ or a single monomer. The ratio of 75\%/25\% was confirmed by Rigby et al. [8] using ENDOR and special triple (ST) spectroscopy. Later, 2D-ESEEM measurements for ${ }^{15} \mathrm{~N}$-labeled P700 [9] and ESEEM analysis of single crystals of PSI [10] reported spin-density distributions of about $90 \% / 10 \%$ and $87.5 \% / 12.5 \%$, respectively. In an extensive study by Mac et al. [94] including ESEEM analysis, isotopic substitution, and numerical simulations of the ESEEM data, it was shown that the spin density of $\mathrm{P} 700^{+}$is completely localized at one Chl a molecule, which interacts with the surrounding protein environment via axial ligation or hydrogen bonds. The complete localization was also reported by Lubitz and co-workers [95] employing ENDOR spectroscopy and site-directed mutation on P700. According to this work, the spin density is localized at the $\mathrm{P}_{\mathrm{B}}$ co-factor. Later, theoretical calculations by Plato et al. [96] confirmed the asymmetric spin-density distribution in the 
inner pair of PSI. The ratio was found to be $75 \% / 25 \%$ in favor of $\mathrm{P}_{\mathrm{B}}$. It was also proposed that this asymmetry is not caused by the structural difference of isolated $\mathrm{P}_{\mathrm{B}}(\mathrm{Chl} a)$ and $\mathrm{P}_{\mathrm{A}}\left(\mathrm{Chl} a^{\prime}\right)$, but by the interaction of the co-factors with the protein environment. This assumption was verified by calculating molecular models including a different number of amino acid residues from the surrounding protein environment. This showed an increasing degree of the spin-density asymmetry for larger molecular models. In case of photo-CIDNP MAS NMR experiments, measured ${ }^{13} \mathrm{C}$ [97] and ${ }^{15} \mathrm{~N}$ [91] signals were assigned to a single Chl $a$ molecule (presumably $\mathrm{P}_{\mathrm{B}}$ ) and to a dimeric donor having an asymmetric spindensity distribution between two unperturbed Chl $a$ co-factors, respectively. Interestingly, in Ref. [98] very different spin-density values were found at Chl nitrogens from spinach (Spinacia oleracea) and aquatic plant duckweed (Spirodella oligorrhiza) pointing to the fact that spin-density distribution could be organism dependent. Despite much effort put into the identification and quantification of spin-density distributions in inner pairs of PSI, the degree of asymmetry still remains ambiguous.

For the BRC of Rhodobacter viridis, an asymmetric spin-density distribution of about $66 \% / 33 \%$ in favor of the $\mathrm{D}_{\mathrm{A}}$ co-factor was reported in Ref. [5]. In case of Rhodobacter sphaeroides, fully-assigned EPR and ENDOR spectra were not presented for a long time. Therefore, many different spin-density ratios were proposed (for an overview of early results, see Refs. [6] and [11]). First fully-assigned ENDOR and TRIPLE spectra of Rhodobacter sphaeroides R-26 in single crystals were presented in Ref. [6], predicting the same $66 \% / 33 \%$ distribution as it was found for Rhodobacter viridis. These results were also confirmed by ESEEM measurements conducted for the primary electron donors P870 from Rhodobacter sphaeroides and P960 from Rhodopseudomonas viridis [7]. A somewhat different spin-density ratio of $60 \% / 40 \%$ was reported later in Ref. [99] based on theoretical KS-DFT computations. Photo-CIDNP MAS NMR measurements carried out for the selectively ${ }^{13} \mathrm{C}$-isotope labeled Rhodobacter sphaeroides WT predicted the spin density to be strongly delocalized over the two BChl a molecules of the SP [13]. 
Interestingly, in this work some small amount of the spin density was also detected at an accessory BChl $a$ molecule. It is, however, generally accepted that no spin-density contributions are present at the axial His molecules as the opposite was not observed in experimental investigations conducted [99-101]. Hence, the BRC SP in the radical cation state is a strongly coupled, slightly asymmetric pair of molecules with the largest contribution localized at the $\mathrm{D}_{\mathrm{A}}$ co-factor.

The detailed results of FDE-diab and FDE-ET calculations for inner pair models with different mutual orientation of co-factors are presented in Tabs. S1-S6 in the SI. In these calculations, the diabatic states are chosen such that $\Phi_{1} \equiv\left|(\mathrm{t}) \mathrm{A}^{+} \cdot . .(\mathrm{t}) \mathrm{B}\right\rangle$ and $\Phi_{2} \equiv$ $\left|(\mathrm{t}) \mathrm{A} \cdot . .(\mathrm{t}) \mathrm{B}^{+\bullet}\right\rangle$, where $\mathrm{A}$ is $\mathrm{P}_{\mathrm{A}}, \mathrm{P}_{\mathrm{D} 1}$ or $\mathrm{D}_{\mathrm{A}}$, while $\mathrm{B}$ is $\mathrm{P}_{\mathrm{B}}, \mathrm{P}_{\mathrm{D} 2}$ or $\mathrm{D}_{\mathrm{B}}$. The case of truncated radical cation models in the electronic ground state is summarized in Fig. 4, while the results for their non-truncated counterparts are given in Fig. 5. As can be seen from Fig. 4 (top), inner pairs of PSI, PSII, and BRC show very different spindensity distributions. At $\Delta R_{\perp}=0 \AA$, the ratios are equal to $96 \% / 4 \%, 80 \% / 20 \%$, and $72 \% / 28 \%$ for the inner pairs in PSI, PSII, and the BRC, respectively. Hence, FDE-diab predicts most of the spin-density to be localized at the $\mathrm{P}_{\mathrm{A}}, \mathrm{P}_{\mathrm{D} 1}$, and $\mathrm{D}_{\mathrm{A}}$ co-factors of the corresponding pairs. The results obtained for PSII and the BRC are in a very good agreement with experimental measurements $[6,8]$, where $82 \%$ and $66 \%$ of the spin-density were found to be localized at $\mathrm{P}_{\mathrm{D} 1}$ and $\mathrm{D}_{\mathrm{A}}$, respectively. In case of PSI, however, most of the spin density is expected to be localized at $\mathrm{P}_{\mathrm{B}}$, while the calculated ratio shows the opposite result. This can probably be explained by the inability of FDE-diab to reproduce the correct order of nearly degenerate electronic states in PSI, which are separated by only $0.14 \mathrm{eV}$. The spin-density ratio in the first excited state of the PSI inner pair is equal to $4 \% / 96 \%$ (see Fig. 5) and, thus, shows the anticipated strong localization at $\mathrm{P}_{\mathrm{B}}$. Another reason for the exchanged order of electronic states could be the complete neglect of co-factor-protein interactions in the presented FDE-diab calculations. The results obtained for non-truncated models are generally very similar (see Fig. 5) to those 
for their truncated counterparts. The spin-density distributions of the PSI inner pair do not show considerable changes and have the same ratio at the equilibrium-distance. In cases of PSII and the BRC, the effect of truncation is stronger and leads to changes in the spin-density ratios of about $4 \%$. The resulting equilibrium distance ratios of the PSII and BRC are equal to $76 \% / 24 \%$ and $68 \% / 32 \%$, respectively. Despite the observed discrepancy in the electronic level ordering for the inner pair of PSI, the obtained results show very high predictive power of the FDE-diab method for studying the charge and spin distributions in photosynthetic systems.

As expected, at larger intermolecular separations [see Fig. 4 (top)] spin-density distributions become more and more localized. The complete localization is observed at displacements $\Delta R_{\perp}$ larger than $1.0 \AA$. This behavior is caused by the absolute magnitudes of the electronic coupling decreasing exponentially [102] with the intermolecular separation. Interestingly, the calculated spin-density ratios for PSI, PSII, and BRC show a qualitatively different behavior at shorter distances. The inner pairs of PSI and PSII have more localized distributions at $\Delta R_{\perp}=-0.5 \AA$ than at the equilibrium distances. Contrary to that, the spin-density of the $\mathrm{BRC}$ reaches a ratio of $62 \% / 38 \%$. This, however, is not observed for the non-truncated molecular models, which all feature a larger degree of delocalization at shorter intermolecular distances (see Fig. 5). It was expected that the overlap of the inner pair co-factor densities and, as the result, the spin-density delocalization degree could both be increased by mutual parallel displacement of aromatic macro-cycles. In case of the BRC, this effect can, indeed, be observed for both truncated and non-truncated models as seen from Fig. 4 (bottom) and Fig. 5 (bottom). The degree of delocalization, however, changes by only about $6-7 \%$ under the shifts of $\Delta R_{\|}=0.6 \AA$ relative to the equilibrium distances. Similar calculations carried out for the PSI and PSII SPs do not show systematic trends under modifications of $R_{\|}$. The changes in the PSI spin-density distributions are comparable in magnitude to the case of the BRC, whereas 2-3 times larger changes can be seen for the PSII inner pair. Although the displacement 

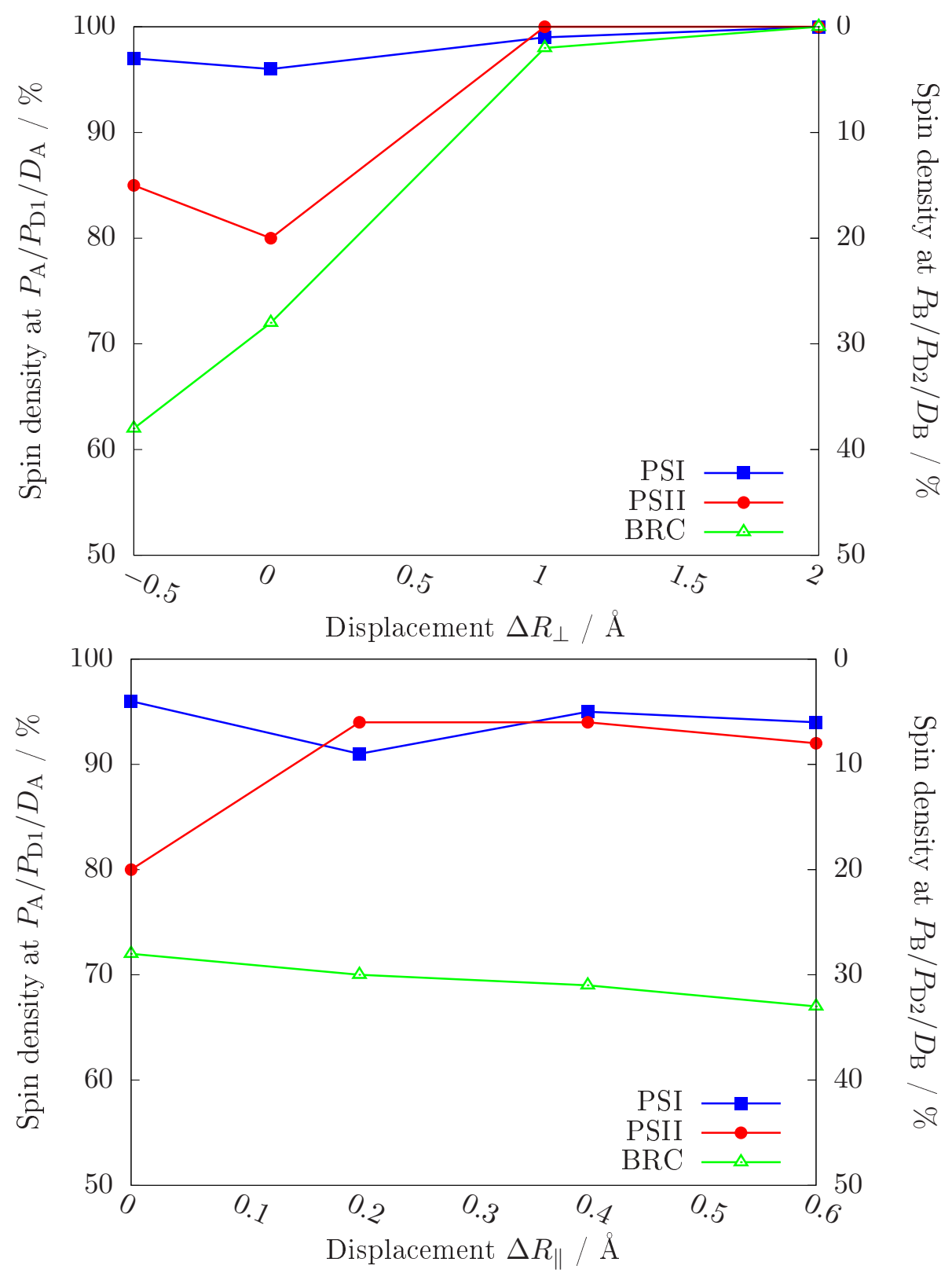

Figure 4: Calculated ground state spin-density ratios for the truncated inner pair models $\left[\mathrm{tP}_{\mathrm{A}} \cdots \mathrm{tP}_{\mathrm{B}}\right]^{+\bullet},\left[\mathrm{tP}_{\mathrm{D} 1} \cdots \mathrm{tP}_{\mathrm{D} 2}\right]^{+\bullet}$ and $\left[\mathrm{tD}_{\mathrm{A}} \cdots \mathrm{tD}_{\mathrm{B}}\right]^{+\bullet}$ from PSI, PSII, and the BRC, respectively. The results are shown for molecular geometries modified by relative vertical $R_{\perp}$ (top) and horizontal $R_{\|}$(bottom) shifts.

of $\Delta R_{\|}=0.2 \AA$ leads to a larger overlap of the aromatic macro-cycles of PSII, the degree of spin-density delocalization decreases by about $14 \%$. Further modifications of $\Delta R_{\|}$show much smaller changes in the spin-density distribution. 

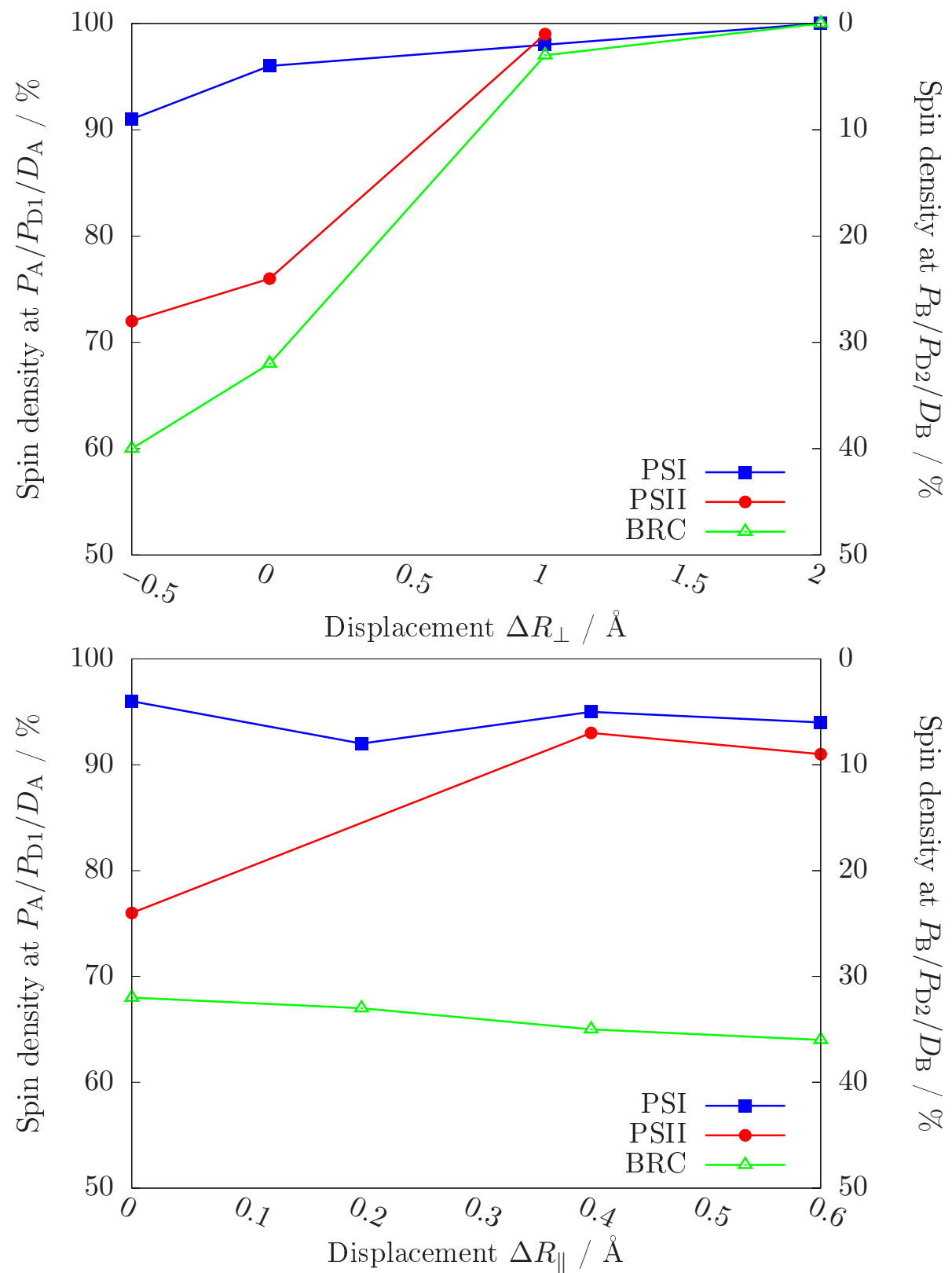

Figure 5: Calculated ground state spin-density ratios for the non-truncated inner pair models $\left[\mathrm{P}_{\mathrm{A}} \cdots \mathrm{P}_{\mathrm{B}}\right]^{+\bullet},\left[\mathrm{P}_{\mathrm{D} 1} \cdots \mathrm{P}_{\mathrm{D} 2}\right]^{+\bullet}$ and $\left[\mathrm{D}_{\mathrm{A}} \cdots \mathrm{D}_{\mathrm{B}}\right]^{+\bullet}$ from PSI, PSII, and the BRC, respectively. The results are shown for molecular geometries modified by relative vertical $R_{\perp}$ (top) and horizontal $R_{\|}$(bottom) shifts. 


\subsection{Influence of the Protein Environment}

To investigate the influence of the nearby protein environment on spin-density distributions, we carried out spin population calculations for a set of small binding pocket models. In these models, all residues within $4.0 \AA$ from the corresponding inner pair were calculated explicitly with FDE, whereas only relaxed MOs of inner (B)Chl molecules were used in FDE-diab computations. For more details on the model setups and structure optimizations, see Sec. 4. The diabatic states were chosen analogously to those considered in Sec. 5.2. FDE-diab calculations for PSII, PSI, and BRC inner pairs in protein binding pockets are presented in Figs. 6 d), 7 d), and 8 d), respectively, whereas a detailed list of the calculated parameters is given in Tab. S7 in the SI. For comparison, in Figs. 7-8 we also present KS-DFT spin populations of a) single (B)Chl a molecule, b) FDE-diab spin populations of inner pairs in vacuum, and c) experimentally measured photo-CIDNP MAS NMR results from Refs. [90, 97].

As can be seen from Figs. 6 a) and b), the spin populations of the dimer $\left[\mathrm{P}_{\mathrm{D} 1} \cdots \mathrm{P}_{\mathrm{D} 2}\right]^{+\bullet}$ in vacuum show distinct differences from the Chl $a$ spin density. The spin distribution in the dimer is shifted towards the ring $A$ showing much larger contributions at carbons $\mathrm{C}-3$ and $\mathrm{C}-3^{2}$ than in the single $\mathrm{Chl} a$ molecule. Also, strong negative spin polarization is obtained at carbon C-2. These differences can probably be explained by the use of the constrained structure-optimization protocol leading to only partially relaxed molecular geometries and, as a result, to artificial spin-density distributions. Contrary to the model in vacuum, the PSII inner pair in the binding protein pocket from Fig. $6 \mathrm{~d}$ ) is fully optimized and, therefore, does not have this artificial asymmetry in the spin distribution. This indicates that the FDE-diab spin density is very sensitive to the quality of the molecular structure used and that more sophisticated protocols of structure optimization are required for accurate calculations of spin populations. As can be seen from Figs. 6 b) and d), the inclusion of the protein environment slightly changes the inner pair spin-density ratio 
from $76 \% / 24 \%$ to $80 \% / 20 \%$ leading to a larger degree of localization at the $\mathrm{P}_{\mathrm{D} 1}$ co-factor. This redistribution leads to a better agreement with the experimental ENDOR results predicting $82 \%$ of spin being localized at $\mathrm{P}_{\mathrm{D} 1}[8]$. Based on these calculations and on experimental measurements reported in Ref. [8], it can be assumed that the remaining spin-density distribution is probably localized at $\mathrm{P}_{\mathrm{D} 2}$ rather than at the axial His. In order to verify this assumption, larger molecular models, which explicitly include two histidine molecules coordinated at the inner pair Chls, need to be calculated with FDEdiab. However, the current implementation of the FDE-diab approach does not allow us to calculate couplings between more than two diabatic states and, therefore, prevents us from conducting these calculations. Comparing experimental photo-CIDNP MAS NMR results and theoretical calculations of protein binding pockets in Figs. 6 c) and d), respectively, it can be seen that most of measured signals are successfully reproduced with FDE-diab. However, somewhat small spin populations are calculated for carbons C-3, C-7, and C13, while only negligible contributions are obtained at C-10 and C-15. This does not directly contradict the photo-CIDNP MAS NMR results depicted in Fig. 6 c) as relative spin populations were not provided in this experimental work [90]. However, it does not support the conslusions drawn by the authors of Ref. [90]. Based on the measured signals, it was proposed in that reference that most of spin density is shifted towards ring $C$ of $\mathrm{Chl} a$ and the spin distribution, thereby, appears to be strongly asymmetric. The calculated spin-density distributions for the inner pair in the binding pocket do not show a strong degree of asymmetry compared to the single Chl $a$ molecule in vacuum as can be seen from Fig. 6 a) and d). This, however, can be a consequence of an insufficiently large size of the binding pocket considered as well as of a complete neglect of axial His MOs in diabatic state construction. These aspects will be investigated in future work, where larger multi-state molecular models will be considered.

The Chl $a$ spin-density populations in the monomer and in the $\left[\mathrm{P}_{\mathrm{A}} \cdots \mathrm{P}_{\mathrm{B}}\right]^{+\bullet}$ dimer are qualitatively different (see Fig. 7). The spin population of the monomer is very similar to 
those calculated earlier for the chlorin and truncated Chl a molecules in Ref. [85]. These spin densities were largely dominated by the highest occupied molecular orbital (HOMO), transforming according to the irreducible representation of point group ${ }^{2} A_{2}$ in case of chlorin and of point group ${ }^{2} A^{\prime \prime}$ for the truncated Chl $a$ molecule [85]. The distribution in Fig. 7 a) is, therefore, closely related to this single ${ }^{2} A_{2}$-like MO. Contrary to that, the spin population in Fig. 7 b) combines features of both HOMO $\left({ }^{2} A_{2}\right.$-like $)$ and HOMO-1 $\left({ }^{2} B_{1^{-}}\right.$ like) MOs of chlorin and is, therefore, strongly mixed. This mixing can be explained by the fact that the molecular structure of $\left[\mathrm{P}_{\mathrm{A}} \cdots \mathrm{P}_{\mathrm{B}}\right]^{+\bullet}$ was only partially relaxed, while a full structure optimization was carried out for the monomer. This assumption is supported by experimental results in Fig. 7 c) as well as by FDE-diab calculations for a fully optimized inner pair in Fig. 7 d). In both cases, the spin density is found to be similar to that for the monomer. The mixing observed for the dimer in vacuum is, therefore, artificial and is, indeed, caused by the limitations of the structure-optimization protocol applied. Comparing results for the inner pair in vacuum and in the protein environment as seen in Figs. 7 b) and d), a redistribution of the spin density from $\mathrm{P}_{\mathrm{A}}$ to $\mathrm{P}_{\mathrm{B}}$ in the latter case can be observed. The resulting spin-density ratio in the binding pocket is equal to $24 \% / 76 \%$ in favor of $\mathrm{P}_{\mathrm{B}}$. This strong localization at the $\mathrm{P}_{\mathrm{B}}$ co-factor is in good agreement with experimental EPR and ENDOR measurements [7-10,12], where $75 \%$ to $100 \%$ of spin density was found at the $\mathrm{P}_{\mathrm{B}}$ co-factor. The calculated ratio in the binding pocket also agrees very well (deviations within 1\%) with previous theoretical computations reported in Ref. [96] and supports the assumption that asymmetry in the inner pair of PSI is induced by the surrounding protein environment. As can be seen from Figs. $7 \mathrm{c}$ ) and $\mathrm{d}$ ), the spin populations of the inner pair in the protein binding pocket are also in good agreement with experimental photo-CIDNP MAS NMR results [97]. Most of the measured spin populations are reproduced by the FDE-diab computations, except for the contributions at $\mathrm{C}-2, \mathrm{C}-8$, and $\mathrm{C}-13^{1}$. Also, FDE-diab predicted small negative spin polarization at carbons C-5 and C-20. These differences could have a number of possible 
explanations such as an insufficient accuracy of the Becke spin population analysis, a not sufficiently accurate molecular structure of the SP, or the comparatively small size of the binding pocket considered. The first issue is investigated in Sec. S3 in the SI, suggesting that the error of the Becke spin population can reach up to 0.04 a.u.. This makes it difficult to reliably reproduce some of the small-intensity signals such as, for example, spin contributions at the four nitrogen atoms of (B)Chl $a$, or at carbon atoms C-5, C-10, C-15, and C-20.

As can be seen from Figs. 8 a) and b), FDE-diab calculations for a monomer of BChl $a$ and for the $\left[\mathrm{D}_{\mathrm{A}} \cdots \mathrm{D}_{\mathrm{B}}\right]^{+\bullet}$ dimer in vacuum do not show pronounced differences in atomic spin populations. Therefore, we can conclude that the chosen constrained structure optimization protocol leads to sufficiently relaxed molecular geometries of the dimer in this case, whereas interaction between co-factors does not introduce a large redistribution of the spin population. The resulting spin-density pattern of the dimer, thereby, appears to be essentially undisturbed compared to the case of single BChl $a$. Inclusion of the nearby protein environment does not introduce qualitative changes in the spin population of the $\left[D_{A} \cdots D_{B}\right]^{+\bullet}$ dimer, as seen in Fig. $8 \mathrm{c}$ ). This observation cannot be validated by comparison with experimental results as, to the best of our knowledge, spin population data is currently unavailable in literature for the BRC. However, the surrounding protein environment does influence the resulting spin-density ratio. Thus, the ratio changes from $68 \% / 32 \%$ for the dimer in vacuum to $93 \% / 7 \%$ for the SP in the protein binding pocket, showing much stronger localization at the $\mathrm{D}_{\mathrm{A}}$ co-factor in the latter case. This result is rather surprising taking into account that ENDOR and TRIPLE experiments [6] as well as previous KS-DFT calculations [99] predict $66 \%$ and $60 \%$ of spin density to be localized at $\mathrm{D}_{\mathrm{A}}$, respectively. However, it agrees with photo-CIDNP experiments reporting a strong localization of the spin density in the SP of Rhodobacter sphaeroides [13]. A definite conclusion on the correct spin-density localization in the SP of the BRC cannot be drawn on the basis of the presented results and would require much larger molecular 
models to be considered. 
a) Monomer

b) Dimer
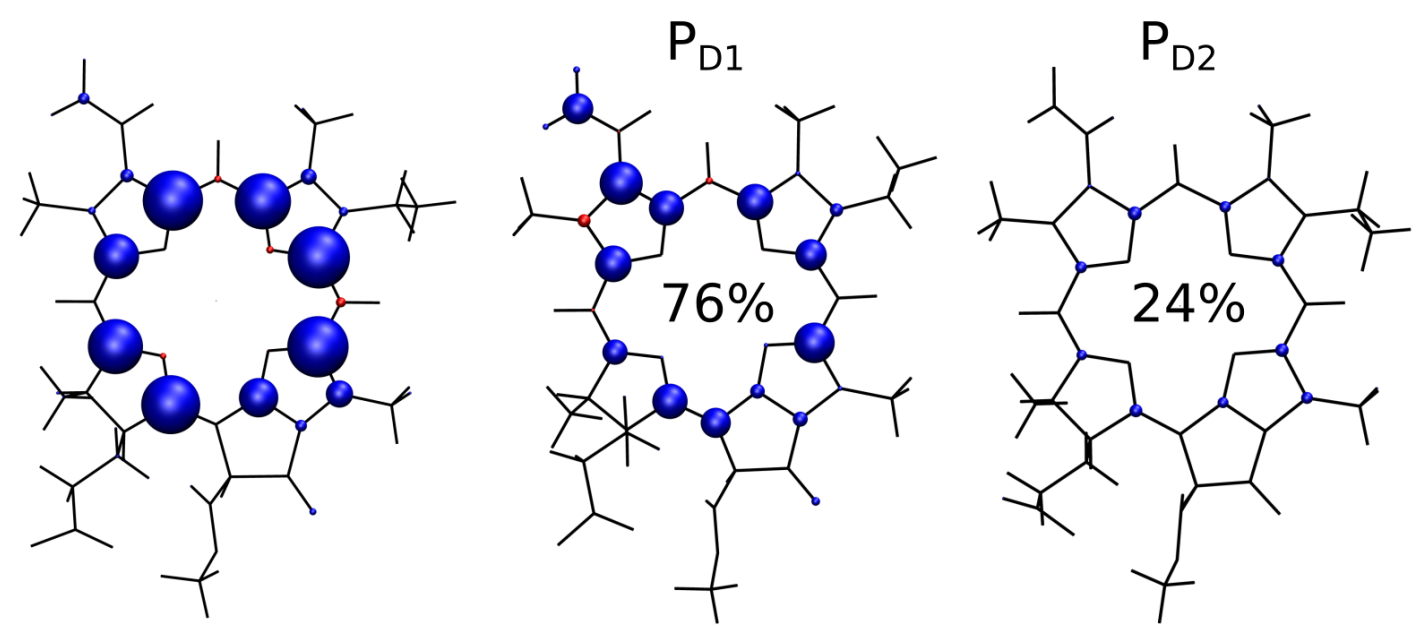

c) Experiment
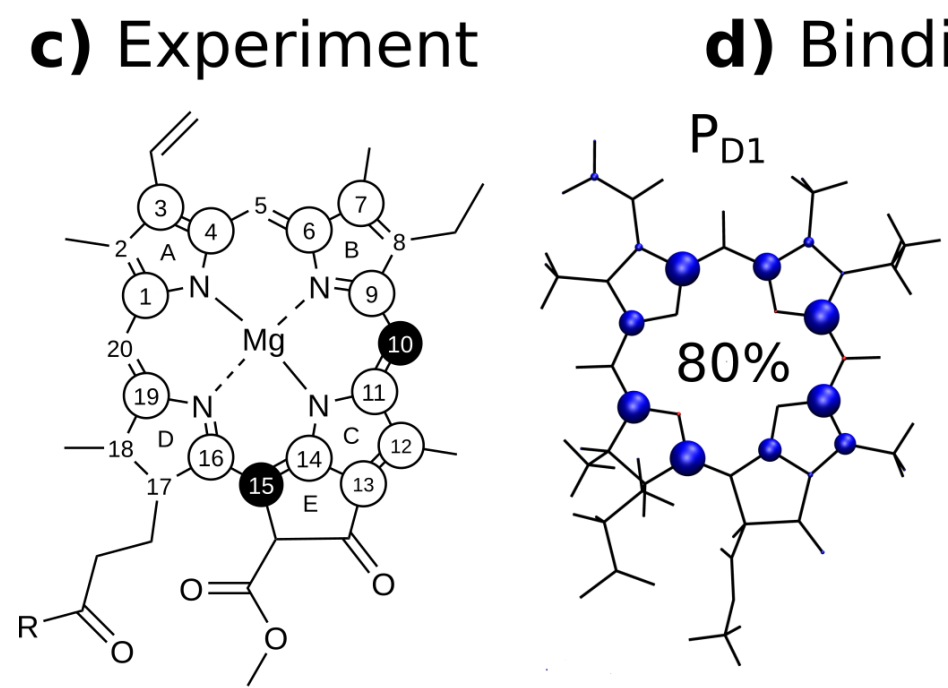

pocket

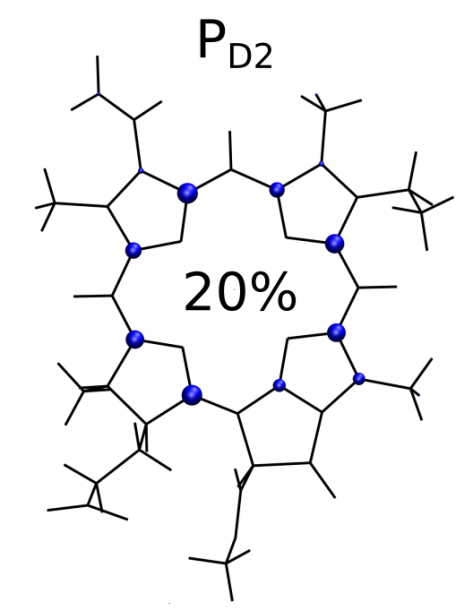

Figure 6: Relative spin-density populations in the inner pair of PSII. The results are presented for calculated electronic ground state spin-density distributions of a) a single Chl $a$ molecule in vacuum, b) a $\left[\mathrm{P}_{\mathrm{D} 1} \cdots \mathrm{P}_{\mathrm{D} 2}\right]^{+\bullet}$ dimer in vacuum, c) experimentally measured in Ref. [90] photo-CIDNP MAS NMR spin densities and for d) a $\left[\mathrm{P}_{\mathrm{D} 1} \cdots \mathrm{P}_{\mathrm{D} 2}\right]^{+\bullet}$ dimer in a protein binding pocket of $R=4 \AA$. Calculated positive and negative contributions are shown by blue and red circles, respectively. The radii of these circles are equally scaled by a factor of 20.0 (for visualization purposes). For dimeric systems, spin-density contributions per molecule are given in percent. White and black cirles indicate positive and negative photo-CIDNP MAS NMR intensities, respectively. 
a) Monomer

b) Dimer
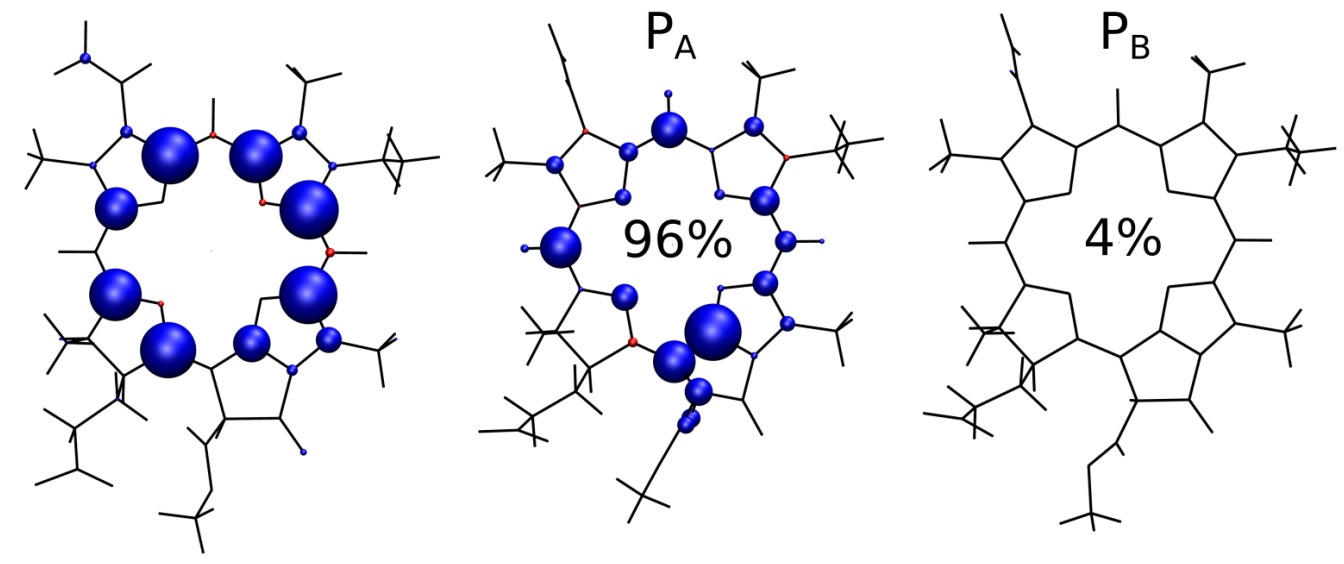

c) Experiment
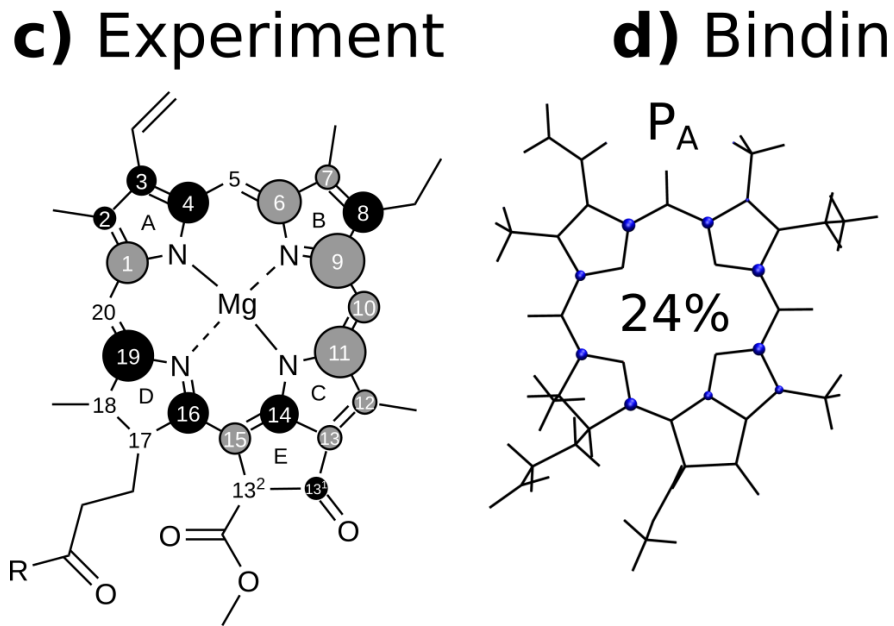

pocket

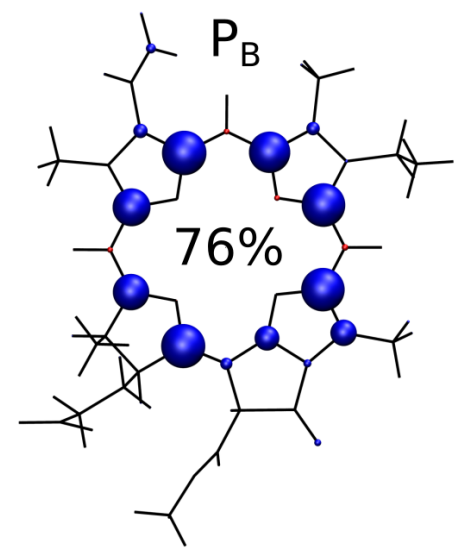

Figure 7: Relative spin-density populations in the inner pair of PSI. The results are presented for calculated electronic ground state spin-density distributions of a) a single Chl $a$ molecule in vacuum, b) a $\left[\mathrm{P}_{\mathrm{A}} \cdots \mathrm{P}_{\mathrm{B}}\right]^{+\bullet}$ dimer in vacuum, c) experimentally measured in Ref. [97] photo-CIDNP MAS NMR spin densities and for d) a $\left[\mathrm{P}_{\mathrm{A}} \cdots \mathrm{P}_{\mathrm{B}}\right]^{+\bullet}$ dimer in a protein binding pocket of $R=4 \AA$. Calculated positive and negative contributions are shown by blue and red circles, respectively. The radii of these circles are equally scaled by a factor of 20.0 (for visualization purposes). For dimeric systems, spin-density contributions per molecule are given in percent. Black and grey cirles indicate negative photo-CIDNP MAS NMR intensities unequivocally assigned and assigned to a few carbon atoms, respectively. 
a) Monomer

b) Dimer
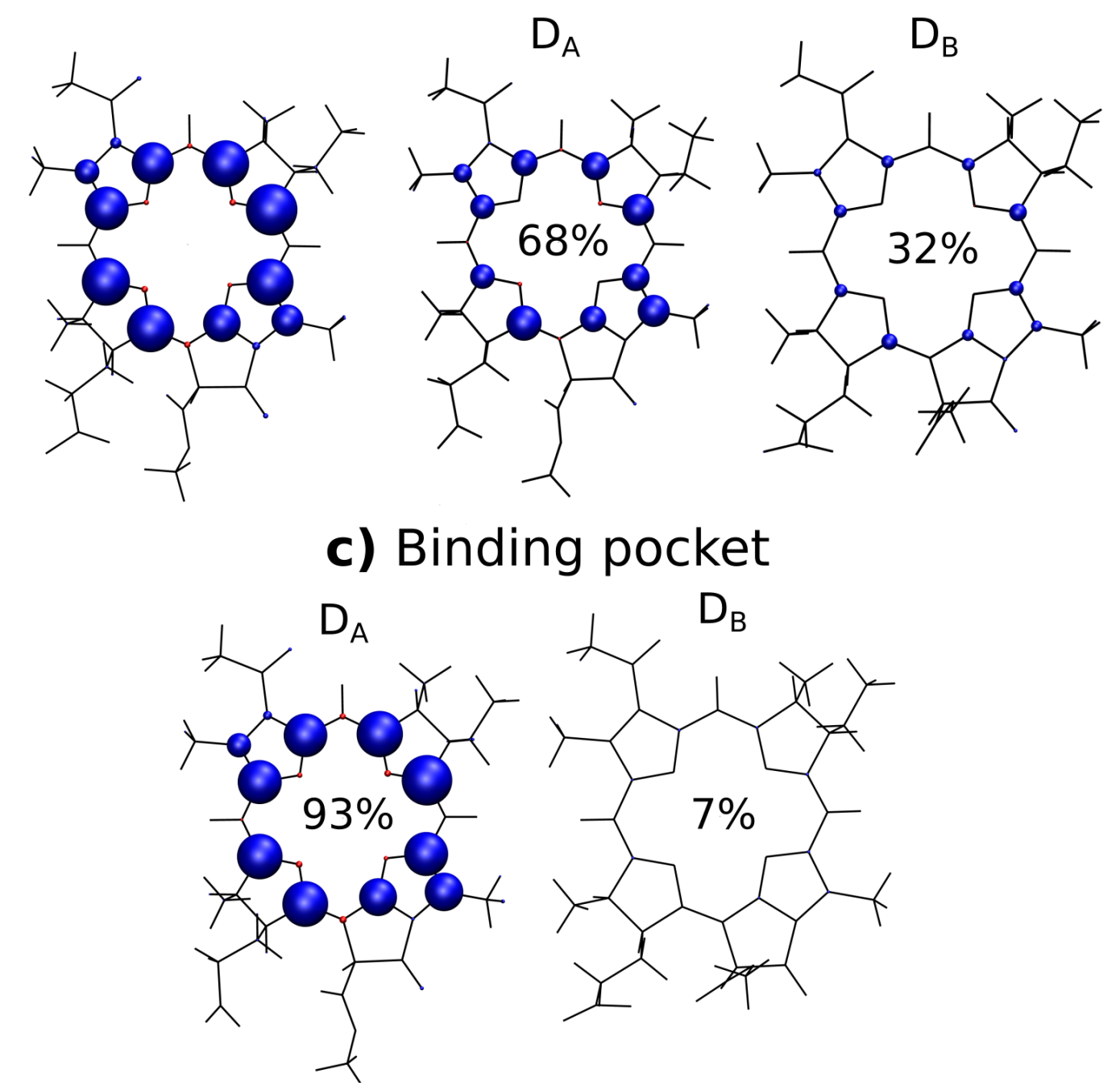

Figure 8: Relative spin-density populations in the SP of the BRC. The results are presented for calculated electronic ground state spin-density distributions of a) a single BChl $a$ molecule in vacuum, b) a $\left[\mathrm{D}_{\mathrm{A}} \cdots \mathrm{D}_{\mathrm{B}}\right]^{+\bullet}$ dimer in vacuum, and c) a $\left[\mathrm{D}_{\mathrm{A}} \cdots \mathrm{D}_{\mathrm{B}}\right]^{+\bullet}$ dimer in a protein binding pocket of $R=4 \AA$. Calculated positive and negative contributions are shown by blue and red circles, respectively. The radii of these circles are equally scaled by a factor of 20.0 (for visualization purposes). For dimeric systems, spin-density contributions per molecule are given in percent. 


\section{Conclusions}

In this work, we presented an application of the recently developed FDE-diab approach to RC models from PSII, PSI, and purple bacteria. This metodology allowed us to avoid the consequences of the DFT overdelocalization error in the intermolecular regime and to reliably calculate spin distributions and electronic couplings for a number of RC models.

The spin-density ratios calculated for the isolated inner pair were equal to about $80 \% / 20 \%$ and $76 \% / 24 \%$ in favor of the $\mathrm{P}_{\mathrm{D} 1}$ co-factor for the trucated and non-truncated models, respectively. Inclusion of the nearby protein environment led to even more delocalized distribitions with the spin-density ratio of $65 \% / 35 \%$ in favor of $\mathrm{P}_{\mathrm{D} 1}$. These results are in good agreement with the experimental ENDOR measurements predicting $82 \%$ of spin to be localized on $\mathrm{P}_{\mathrm{D} 1}$ [8]. Taking into account large electronic couplings between the inner pair co-factors, we can assume that the remaining spin density is probably localized at the $\mathrm{P}_{\mathrm{D} 2}$ molecule. This, however, contradicts the hinge-type model for the $\mathrm{RC}$ in PSII suggested in Ref. [91] and suggests the delocalization of the spin-density distribution between $\mathrm{P}_{\mathrm{D} 1}$ and the axial His molecule. Calculations of spin-density ratios between two axial His molecules and the inner pair require the use of MOs of all four molecules in the diabatic-states construction procedure, and are not possible in terms of two-state models. This requires a generalization of the FDE-diab approach to multiple electronic states. Although most of experimentally measured spin-density populations of the $\mathrm{P}_{\mathrm{D} 1}$ co-factor in PSII $[90,91]$ were successfully reproduced by us in FDE-diab calculations of large protein binding pockets, the proposed strong asymmetry in the spin-density pattern was also not observed.

FDE-diab calculations of isolated inner pair models from PSI predicted about $96 \%$ of spin density being localized at $\mathrm{P}_{\mathrm{A}}$. Although the exact spin-density ratio for the inner pair of PSI is unknown, various experimental measurements (for example, see Refs. [7- 
10, 12]) predict from $75 \%$ to $100 \%$ of the spin density to be localized at the $\mathrm{P}_{\mathrm{B}}$ cofactor. Therefore, the FDE-diab calculations for isolated inner pair models contradict experimental results. This, however, is caused by a complete neglect of the co-factorprotein interactions. Inclusion of the protein environment into FDE-diab calculations changed the spin-density distribution considerably and resulted in the ratio of $24 \% / 76 \%$ in favor of $\mathrm{P}_{\mathrm{B}}$, which is in good agreement with available experimental results. We also managed to reproduce most of experimentally measured $\mathrm{P}_{\mathrm{B}}$ spin populations [97] using the large protein binding pocket model. However, it turned out to be difficult to reliably calculate some small atomic spin contributions.

FDE-diab calculations of SPs in the BRC predicted spin-density ratios of $72 \% / 28 \%$ and $68 \% / 32 \%$ in favor of $\mathrm{D}_{\mathrm{A}}$ for truncated and non-truncated models, respectively. These results are in excellent agreement with the experimentally measured ratio of $66 \% / 33 \%$ [5]. However, FDE-diab calculations for a binding pocket model show a somewhat larger degree of spin-density localization with the ratio of $93 \% / 7 \%$. It is also interesting to note that the calculated spin populations for the isolated $\mathrm{BChl} a$ and the $\mathrm{D}_{\mathrm{A}}$ co-factor from the binding pocket are very similar. This could indicate that the SP in the BRC is essentially unperturbed by the protein environment.

Summarizing the results presented in this work, we conclude that the FDE-diab methodology is a valuable and robust tool for spin-density calculations of large molecular systems. Avoiding the consequences of the DFT overdelocalization error in the intermolecular regime, it allowed us to obtain reliable spin-density distributions for a number of RC models and to gain a deep insight into the asymmetry of the corresponding SPs. Moreover, the spin-density distribution was found to be highly sensitive to structural arrangements of co-factors. Spin-density distributions of RC models as calculated in this work were not accessible before with existing computational techniques. Further extensions of the FDE-diab approach will soon allow us to go beyond the simplistic two-state model and 
access spin densities of even larger molecular models.

\section{Acknowledgments}

We thank Prof. Dr. Jörg Matysik for his helpful comments. D.G.A. acknowledges funding from the European Union's Horizon 2020 research and innovation programme under the Marie Skłodowska-Curie grant agreement no. 835776. 


\section{References}

[1] R. E. Blankenship. Molecular Mechanisms of Photosynthesis. Blackwell Science, Oxford, 2002.

[2] E. Daviso, S. Prakash, A. Alia, P. Gast, J. Neugebauer, G. Jeschke, J. Matysik. The electronic structure of the primary electron donor of reaction centers of purple bacteria at atomic resolution as observed by photo-CIDNP ${ }^{13} \mathrm{C}$ NMR. PNAS, 106 (2009) 22281-22286.

[3] M. Najdanova, G. J. Janssen, H. J. M. de Groot, J. Matysik, A.Alia. Analysis of electron donors in photosystems in oxygenic photosynthesis by photo-CIDNP MAS NMR. J. Photochem. Photobiol. B, 152 (2015) 261-271.

[4] J. Deisenhofer, O. Epp, I. Sinning, H. Michel. Crystallographic Refinement at 2.3 A Resolution and Refined Model of the Photosynthetic Reaction Centre from Rhodopseudomonas viridis. J. Mol. Biol., 246 (1995) 429-457.

[5] F. Lendzian, W. Lubitz, H. Scheer, A. J. Hoff, M. Plato, E.Tränkle, K. Möbius. ESR, ENDOR and TRIPLE resonance studies of the primary donor radical cation $\mathrm{P}_{960}^{+}$in the photosynthetic bacterium Rhodopseudomonas viridis. Chem. Phys. Lett., 148 (1988) 377-385.

[6] F. Lendzian, M. Huberb, R. A. Isaacson, B. Endeward, M.Plato, B. Bönigk, K. Möbius, W. Lubitz, G. Feher. The electronic structure of the primary donor cation radical in Rhodobacter sphaeroides R-26: ENDOR and TRIPLE resonance studies in single crystals of reaction centers. Biochim. Biophys. Acta, 1183 (1993) $139-160$.

[7] I. H. Davis, P. Heathcote, D. J. MacLachlan, M. C. W. Evans. Modulation analysis of the electron spin echo signals of in vivo oxidised primary donor $14 \mathrm{~N}$ chlorophyll 
centres in bacterial, P870 and P960, and plant Photosystem I, P700, reaction centres. Biochim. Biophys. Acta, 1143 (1993) 183-189.

[8] S. E. J. Rigby, J. H. A. Nugent, P. J. O’Malley. ENDOR and Special Triple Resonance Studies of Chlorophyll Cation Radicals in Photosystem 2. Biochemistry, 33 (1994) 10043-10050.

[9] H. Käß, W. Lubitz. Evaluation of 2D-ESEEM data of ${ }^{15} \mathrm{~N}$-labeled radical cations of the primary donor P700 in photosystem I and chlorophyll a. Chem. Phys. Lett., 251 (1996) 193-203.

[10] H. Käß, P. Fromme, W. Lubitz. Quadrupole parameters of nitrogen nuclei in the cation radical $\mathrm{P}_{700}^{++}$determined by ESEEM of single crystals of phtosystem I. Chem. Phys. Lett., 257 (1996) 197-206.

[11] M. Huber. On the electronic structure of the primary electron donor in bacterial photosynthesis — the bacteriochlorophyll dimer as viewed by EPR/ENDOR methods. Photosynth. Res., 52 (1997) 1-26.

[12] J. Breton, E. Nabedryk, W. Leibl. FTIR Study of the Primary Electron Donor of Photosystem I (P700) Revealing Delocalization of the Charge in P700 ${ }^{+}$and Localization of the Triplet Character in ${ }^{3}$ P700. Biochemistry, 38 (1999) 11585-11592.

[13] E. A. M. Schulten, J. Matysik, A. Alia, S. Kiihne, J. Raap, J. Lugtenburg, P. Gast, A. J. Hoff, H. J. M. de Groot. ${ }^{13}$ C MAS NMR and Photo-CIDNP Reveal a Pronounced Asymmetry in the Electronic Ground State of the Special Pair of Rhodobacter sphaeroides Reaction Centers. Biochemistry, 41 (2002) 8708-8717.

[14] S. Prakash, A. Alia, P. Gast, G. Jeschke, H. J. M. de Groot, J. Matysik. Photochemically induced dynamic nuclear polarisation in entire bacterial photosynthetic units observed by ${ }^{13} \mathrm{C}$ magic-angle spinning NMR. J. Mol. Struct., 661 (2003) 625-633. 
[15] G. J. Janssen, E. Daviso, M. van Son, H. J. M. de Groot, A. Alia, J. Matysik. Observation of the solid-state photo-CIDNP effect in entire cells of cyanobacteria Synechocystis. Photosynth. Res., 104 (2010) 275-282.

[16] G. J. Janssen, P. Bielytskyi, D. G. Artiukhin, J. Neugebauer, H. J. M. de Groot, J. Matysik, A. Alia. Photochemically induced dynamic nuclear polarization NMR on photosystem II: donor cofactor observed in entire plant. Sci. Rep., 8 (2018) 17853.

[17] M. Kaupp, M. Bühl, V. G. Malkin, Eds. Calculation of NMR and EPR parameters: Theory and Applications. WILEY-VCH Verlag GmbH \& Co. KGaA, Weinheim, 2004.

[18] D. Dolphin, R. H. Felton, D. C. Borg, J. Fajer. Isoporphyrins. J. Am. Chem. Soc., 92 (1970) 743-745.

[19] J. Fajer, M. S. Davis. Electron spin resonance of porphyrin $\pi$ cations and anions. In D. Dolphin, Ed., The Porphyrins, Volume IV, Physical Chemistry, Part B. Academic Press, New York, 1979.

[20] H. Käß, E. Bittersmann-Weidlich, L.-E. Andréasson, B. Bönigk, W. Lubitz. ENDOR and ESEEM of the $15 \mathrm{~N}$ labelled radical cations of chlorophyll a and the primary donor P700 in photosystem I. Chem. Phys., 194 (1995) 419-432.

[21] W. Lubitz. Pulse EPR and ENDOR studies of light-induced radicals and triplet states in photosystem II of oxygenic photosynthesis. Phys. Chem. Chem. Phys., 4 (2002) 5539-5545.

[22] F. Lendzian, M. Huber, R. A. Isaacson, B. Endeward, M. Plato, B. Bönigk, K. Möbius, W. Lubitz, G. Feher. The electronic structure of the primary donor cation radical in Rhodobacter sphaeroides R-26: ENDOR and TRIPLE resonance studies in single crystals of reaction centers. Biochim. Biophys. Acta, 1183 (1993) $139-160$. 
[23] P. Mori-Sánchez, A. J. Cohen, W. Yang. Many-electron self-interaction error in approximate density functionals. J. Chem. Phys., 125 (2006) 201102.

[24] J. Gräfenstein, E. Kraka, D. Cremer. Effect of the self-interaction error for threeelectron bonds: On the development of new exchange-correlation functionals. Phys. Chem. Chem. Phys., 6 (2004) 1096-1112.

[25] A. J. Cohen, P. Mori-Sánchez, W. Yang. Development of exchange-correlation functionals with minimal many-electron self-interaction error. J. Chem. Phys., 126 (2007) 191109.

[26] A. J. Cohen, P. Mori-Sánchez, W. Yang. Insights into Current Limitations of Density Functional Theory. Science, 321 (2008) 792-794.

[27] A. Solovyeva, M. Pavanello, J. Neugebauer. Spin densities from subsystem densityfunctional theory: Assessment and application to a photosynthetic reaction center complex model. J. Chem. Phys., 136 (2012) 194104.

[28] D. G. Artiukhin, J. Neugebauer. Frozen-density embedding as a quasi-diabatization tool: Charge-localized states for spin-density calculations. J. Chem. Phys., 148 (2018) 214104.

[29] J. Conradie, A. Ghosh. DFT Calculations on the Spin-Crossover Complex Fe(salen)(NO): A Quest for the Best Functional. J. Phys. Chem. B, 111 (2007) $12621-12624$.

[30] K. Boguslawski, C. R. Jacob, M. Reiher. Can DFT Accurately Predict Spin Densities? Analysis of Discrepancies in Iron Nitrosyl Complexes. J. Chem. Theory Comput., 7 (2011) 2740-2752.

[31] K. Boguslawski, K. H. Marti, Ö. Legeza, M. Reiher. Accurate ab Initio Spin Densities. J. Chem. Theory Comput., 8 (2012) 1970. 
[32] V. Riffet, D. Jacquemin, E. Cauët, G. Frison. Benchmarking DFT and TD-DFT Functionals for the Ground and Excited States of Hydrogen-Rich Peptide Radicals. J. Chem. Theory Comput., 10 (2014) 3308-3318.

[33] J. P. Perdew, Alex Zunger. Self-interaction correction to density-functional approximations for many-electron systems. Phys. Rev. B, 23 (1982) 5048-5079.

[34] Aron J. Cohen, Paula Mori-Sánchez, Weitao Yang. Insights into Current Limitations of Density Functional Theory. Science, 321 (2008) 792-794.

[35] T. A. Wesołowski, A. Warshel. Frozen density functional approach for ab initio calculations of solvated molecules. J. Phys. Chem., 97 (1993) 8050-8053.

[36] M. Pavanello, J. Neugebauer. Modelling charge transfer reactions with the frozen density embedding formalism. J. Chem. Phys., 135 (2011) 234103.

[37] M. Pavanello, T. Van Voorhis, L. Visscher, J. Neugebauer. An accurate and linearscaling method for calculating charge-transfer excitation energies and diabatic couplings. J. Chem. Phys., 138 (2013) 054101.

[38] A. Solovyeva, M. Pavanello, J. Neugebauer. Describing long-range charge-separation processes with subsystem density-functional theory. J. Chem. Phys., 140 (2014) 164103.

[39] P. Ramos, M. Papadakis, M. Pavanello. Performance of Frozen Density Embedding for Modeling Hole Transfer Reactions. J. Phys. Chem. B, 119 (2015) 7541-7557.

[40] T. Van Voorhis, T. Kowalczyk, B. Kaduk, L.-P. Wang, C.-L. Cheng, Q. Wu. The diabatic picture of electron transfer, reaction barriers, and molecular dynamics. Annu. Rev. Phys. Chem., 61 (2010) 149-170.

[41] C.-P. Hsu. The Electronic Couplings in Electron Transfer and Excitation Energy Transfer. Acc. Chem. Res., 42 (2009) 509-518. 
[42] M. D. Newton. Quantum chemical probes of electron-transfer kinetics: the nature of donor-acceptor interactions. Chem. Rev., 91 (1991) 767-792.

[43] A. Migliore. Nonorthogonality Problem and Effective Electronic Coupling Calculation: Application to Charge Transfer in $\pi$-Stacks Relevant to Biochemistry and Molecular Electronics. J. Chem. Theory Comput., 7 (2011) 1712-1725.

[44] F. Plasser, H. Lischka. Semiclassical dynamics simulations of charge transport in stacked $\pi$-systems. J. Chem. Phys., 134 (2011) 034309.

[45] Y. Umena, K. Kawakami, J.-R. Shen, N. Kamiya. Crystal structure of oxygenevolving photosystem II at a resolution of 1.9 A. Nature, 473 (2011) 55-61.

[46] P. Jordan, P. Fromme, H. T. Witt, O. Klukas, W. Saenger, N. Krauss. Threedimensional structure of cyanobacterial photosystem I at $2.5 \AA$ resolution. Nature, 411 (2001) 909-917.

[47] A. Camara-Artigas, D. Brune, J. P. Allen. Interactions between lipids and bacterial reaction centers determined by protein crystallography. PNAS, 99 (2002) 1105511060.

[48] K. N. Ferreira, T. M. Iverson, K. Maghlaoui, J. Barber, S. Iwata. Architecture of the Photosynthetic Oxygen-Evolving Center. Science, 303(5665) (2004) 1831-1838.

[49] T. Watanabe, M. Kobayashi, A. Hongu, M. Nakazato, T. Hiyama, N. Murata. Evidence that a chlorophyll $a^{\prime}$ dimer constitutes the photochemical reaction centre 1 (P700) in photosynthetic apparatus. FEBS Lett., 191 (1985) 252-256.

[50] A. N. Webber, W. Lubitz. P700: the primary electron donor of photosystem I. Biochim. Biophys. Acta, 1507 (2001) 61-79.

[51] T.A. Wesołowski. Computational Chemistry: Reviews of Current Trends, Volume 10, Chapter One-Electron Equations for Embedded Electron Density: Challenge for The- 
ory and Practical Payoffs in Multi-Level Modelling of Complex Polyatomic Systems, p. 1-82. World Scientific, 2006.

[52] A. Migliore, S. Corni, D. Versano, M. L. Klein, R. Di Felice. First Principles Effective Electronic Couplings for Hole Transfer in Natural and Size-Expanded DNA. J. Phys. Chem. B, 113 (2009) 9402-9415.

[53] Q. Wu, T. Van Voorhis. Direct optimization method to study constrained systems within density-functional theory. Phys. Rev. A, 72 (2005) 024502.

[54] H. Oberhofer, J. Blumberger. Electronic coupling matrix elements from charge constrained density functional theory calculations using a plane wave basis set. J. Chem. Phys., 133 (2010) 244105.

[55] P.-O. Löwdin. Quantum theory of many-particle systems. I. Physical interpretations by means of density matrices, natural spin-orbitals, and convergence problems in the method of configurational interaction. Phys. Rev., 97 (1955) 1474-1489.

[56] I. Mayer. Simple Theorems, Proofs, and Derivations in Quantum Chemistry. Kluwer Academic/Plenum, 2003.

[57] B. Kaduk, T. Kowalczyk, T. Van Voorhis. Constrained Density Functional Theory. Chem. Rev., 112 (2012) 321.

[58] T. A. Wesołowski, J. Weber. Kohn-Sham equations with constrained electron density: An iterative evaluation of the ground-state electron density of interacting molecules. Chem. Phys. Lett., 248 (1996) 71-76.

[59] A. J. W. Thom, M. Head-Gordon. Hartree-Fock solutions as a quasidiabatic basis for nonorthogonal configuration interaction. J. Chem. Phys., 131 (2009) 124113.

[60] S. Efrima, M. Bixon. Vibrational effects in outer-sphere electron-transfer reactions in polar media. Chem. Phys., 13 (1976) 447-460. 
[61] H. M. Berman, J. Westbrook, Z. Feng, G. Gilliland, T. N. Bhat, H. Weissig, I. N. Shindyalov, P. E. Bourne. The Protein Data Bank. Nucleic Acids Res., 28 (2000) $235-242$.

[62] J. M. Word, S. C. Lovell, J. S. Richardson, D. C. Richardson. Asparagine and Glutamine: Using Hydrogen Atom Contacts in the Choice of Side-chain Amide Orientation. J. Mol. Biol., 285 (1999) 1735-1747.

[63] A.D. MacKerell, M. Feig, C. L. Brooks. Extending the treatment of backbone energetics in protein force fields: Limitations of gasphase quantum mechanics in reproducing protein conformational distributions in molecular dynamics simulations. J. Comput. Chem., 25 (2004) 1400-1415.

[64] A. D. MacKerell, D. Bashford, M. Bellott, R. L. Dunbrack, J. D. Evanseck, M. J. Field, S. Fischer, J. Gao, H. Guo, S. Ha, D. Joseph-McCarthy, L. Kuchnir, K. Kuczera, F. T. K. Lau, C. Mattos, S. Michnick, T. Ngo, D. T. Nguyen, B. Prodhom, W. E. Reiher, B. Roux, M. Schlenkrich, J. C. Smith, R. Stote, J. Straub, M. Watanabe, J. Wiórkiewicz-Kuczera, D. Yin, M. Karplus. All-atom empirical potential for molecular modeling and dynamics studies of proteins. The Journal of Physical Chemistry $B, 102$ (1998) 3586-3616.

[65] F. H. Allen, O. Kennard, D. G. Watson, L. Brammer, A. G. Orpen, R. Taylor. Tables of bond lengths determined by X-ray and neutron diffraction. Part 1. Bond lengths in organic compounds. J. Chem. Soc., Perkin Trans. 2, 1987) S1.

[66] F. Neese. The ORCA program system. Comput. Mol. Sci., 2 (2012) 73-78.

[67] Elemental Data and Radii sheet published by The Cambridge Crystallographic Data Centre, URL: https://www.ccdc.cam.ac.uk/support-andresources/ccdcresources/Elemental_Radii.xlsx, access date: May 7, 2019. 
[68] B. Cordero, V. Gómez, A. E. Platero-Prats, M. Revés, J. Echeverría, E. Cremades, F. Barragan, S. Alvarez. Covalent radii revisited. Dalton Trans., 0 (2008) 28322838.

[69] A. D. Becke. Density-functional exchange-energy approximation with correct asymptotic behavior. Phys. Rev. A, 38(6) (1988) 3098-3100.

[70] J. P. Perdew. Density-functional approximation for the correlation energy of the inhomogeneous electron gas. Phys. Rev. B, 33 (1986) 8822-8824.

[71] A. Schäefer, H. Horn, R. Ahlrichs. Fully optimized contracted Gaussian basis sets for atoms Li to Kr. J. Chem. Phys., 97 (1992) 2571-2577.

[72] A. Schäfer, C. Huber, R. Ahlrichs. Fully optimized contracted Gaussian basis sets of triple zeta valence quality for atoms Li to Kr. J. Chem. Phys., 100 (1994) 5829.

[73] S. Grimme, J. Antony, S. Ehrlich, H. Krieg. A consistent and accurate ab initio parametrization of density functional dispersion correction (DFT-D) for the 94 elements H-Pu. J. Chem. Phys., 132 (2010) 154104.

[74] S. Grimme, S. Ehrlich, L. Goerigk. Effect of the damping function in dispersion corrected density functional theory. J. Comput. Chem., 32 (2011) 1456-1465.

[75] M. Gaus, Q. Cui, M. Elstner. DFTB3: Extension of the Self-Consistent-Charge Density-Functional Tight-Binding Method (SCC-DFTB). J. Chem. Theory Comput., 7(4) (2011) 931-948.

[76] Amsterdam density functional program, Theoretical Chemistry, Vrije Universiteit, Amsterdam, URL: http://www.scm.com, access date: 13 January 2018.

[77] M. Gaus, A. Goez, M. Elstner. Parametrization and Benchmark of DFTB3 for Organic Molecules. J. Chem. Theory Comput., 9(1) (2013) 338-354. 
[78] M. Kubillus, T. Kubar, M. Gaus, J. Rezac, M. Elstner. Parameterization of the DFTB3 Method for Br, Ca, Cl, F, I, K, and Na in Organic and Biological Systems. J. Chem. Theory Comput., 11(1) (2015) 332-342.

[79] C. R. Jacob, S. M. Beyhan, R. E. Bulo, A. S. P. Gomes, A. W. Götz, K. Kiewisch, J. Sikkema, L. Visscher. PyADF - A scripting framework for multiscale quantum chemistry. J. Comput. Chem., 32 (2011) 2328-2338.

[80] E. van Lenthe, E. J. Baerends. Optimized Slater-type basis sets for the elements 1-118. J. Comput. Chem., 24 (2003) 1142-1156.

[81] J. P. Perdew, J. A. Chevary, S. H. Vosko, K. A. Jackson, M. R. Pederson, D. J. Singh, C. Fiolhais. Atoms, molecules, solids, and surfaces: Applications of the generalized gradient approximation for exchange and correlation. Phys. Rev. B, 46 (1992) 6671.

[82] J. P. Perdew, Y. Wang. Electronic Structure of Solids'91. Academie, Berlin, 1991.

[83] H. Lee, C. Lee, R. G. Parr. Conjoint gradient correction to the Hartree-Fock kineticand exchange-energy density functionals. Phys. Rev. A, 44 (1991) 768-771.

[84] A. Lembarki, H. Chermette. Obtaining a gradient-corrected kinetic-energy functional from the Perdew-Wang exchange functional. Phys. Rev. A, 50 (1994) 5328-5331.

[85] D. G. Artiukhin, C. J. Stein, M. Reiher, J. Neugebauer. Quantum Chemical Spin Densities for Radical Cations of Photosynthetic Pigment Models. Photochem. Photobiol., 93 (2017) 815-833.

[86] O. Treutler, R. Ahlrichs. Efficient molecular numerical integration schemes. J. Chem. Phys., 102 (1995) 346.

[87] M. Franchini, P. H. T. Philipsen, L. Visscher. The Becke Fuzzy Cells Integration Scheme in the Amsterdam Density Functional Program Suite. J. Comput. Chem., 34 (2013) 1819. 
[88] J. P. Unsleber, T. Dresselhaus, K. Klahr, D. Schnieders, M. Böckers, D. Barton, J. Neugebauer. Serenity: A subsystem quantum chemistry program. J. Comput. Chem., 39 (2018) 788-798.

[89] A. Diller, A. Alia, E. Roy, P. Gast, H. J. van Gorkom, J. Zaanen, H. J. M. de Groot, C. Glaubitz, J. Matysik. Photo-CIDNP solid-state NMR on Photosystems I and II: what makes P680 special? Photosynth. Res., 84 (2005) 303-308.

[90] J. Matysik, A. Alia, P. Gast, H. J. van Gorkom, A. J. Hoff, H. J. M. de Groot. Photochemically induced nuclear spin polarization in reaction centers of photosystem II observed by ${ }^{13} \mathrm{C}$-solid-state NMR reveals a strongly asymmetric electronic structure of the $\mathrm{P}_{680}^{++}$primary donor chlorophyll. PNAS, 97 (2000) 9865-9870.

[91] A. Diller, E. Roy, P. Gast, H. J. van Gorkom, H. J. M. de Groot, C. Glaubitz, G. Jeschke, J. Matysik, A. Alia. ${ }^{15} \mathrm{~N}$ photochemically induced dynamic nuclear polarization magic-angle spinning NMR analysis of the electron donor of photosystem II. PNAS, 104 (2007) 12767-12771.

[92] J. R. Norris, R. A. Uphaus, H. L. Crespi, J. J. Katz. Electron Spin Resonance of Chlorophyll and the Origin of Signal I in Photosynthesis. PNAS, 68 (1971) 625-628.

[93] J. R. Norris, H. Scheer, M. E. Druyan, J. J. Katz. An Electron-Nuclear Double Resonance (ENDOR) Study of the Special Pair Model for Photo-Reactive Chlorophyll in Photosynthesis. PNAS, 71 (1974) 4897-4900.

[94] M. Mac, N. R. Bowlby, G. T. Babcock, J. McCracken. Monomeric Spin Density Distribution in the Primary Donor of Photosystem I as Determined by Electron Magnetic Resonance: Functional and Thermodynamic Implications. J. Am. Soc., 120 (1998) 13215-13223.

[95] L. Krabben, E. Schlodder, R. Jordan, D. Carbonera, G. Giacometti, H. Lee, A. N. Webber, W. Lubitz. Influence of the Axial Ligands on the Spectral Properties of 
P700 of Photosystem I. A Study of Site-Directed Mutants. Biochemistry, 39(42) (2000) 13012-13025.

[96] M. Plato, N. Krauß, P. Fromme, W. Lubitz. Molecular orbital study of the primary electron donor P700 of photosystem I based on a recent X-ray single crystal structure analysis. Chem. Phys., 294(3) (2003) 483-499.

[97] A. Alia, E. Roy, P. Gast, H. J. van Gorkom, H. J. M. de Groot, G. Jeschke, J. Matysik. Photochemically induced dynamic nuclear polarization in photosystem I of plants observed by ${ }^{13} \mathrm{C}$ magic-angle spinning NMR. J. Am. Chem. Soc., 126 (2004) 12819 12826.

[98] G.J. Janssen, E. Roy, J. Matysik, A. Alia. ${ }^{15} \mathrm{~N}$ photo-CIDNP MAS NMR to reveal functional heterogeneity in electron donor of different plant organisms. Appl. Magn. Reson., 42 (2012) 57-67.

[99] S. Prakash, A. Alia, P. Gast, H. J. M. de Groot, G. Jeschke, J. Matysik. Magnetic Field Dependence of Photo-CIDNP MAS NMR on Photosynthetic Reaction Centers of Rhodobacter sphaeroides WT. J. Am. Chem. Soc., 127 (2005) 14290-14298.

[100] M. G. Zysmilich, A. McDermott. Photochemically Induced Dynamic Nuclear Polarization in the Solid-State ${ }^{15} \mathrm{~N}$ Spectra of Reaction Centers from Photosynthetic Bacteria Rhodobacter sphaeroides R-26. J. Am. Chem. Soc., 116 (1994) 8362-8363.

[101] M. G. Zysmilich, A. McDermott. Photochemically Induced Nuclear Spin Polarization in Bacterial Photosynthetic Reaction Centers: Assignments of the ${ }^{15} \mathrm{~N}$ SSNMR Spectra. J. Am. Chem. Soc., 118 (1996) 5867-5873.

[102] F. Hernández-Fernández, M. Pavanello, L. Visscher. Effect of metallation, substituents and inter/intra-molecular polarization on electronic couplings for hole transport in stacked porphyrin dyads. Phys. Chem. Chem. Phys., 18 (2016) 21122-21132. 